\title{
Monolithic multigrid method for the coupled Stokes flow and deformable porous medium system
}

\author{
P. Luo ${ }^{\mathrm{a}, *}$, C. Rodrigo ${ }^{\mathrm{b}}$, F.J. Gaspar ${ }^{\mathrm{c}}$, C.W. Oosterlee ${ }^{\mathrm{a}, \mathrm{c}}$ \\ a DIAM, Delft University of Technology, The Netherlands \\ b Department of Applied Mathematics, University of Zaragoza, Spain \\ c CWI, Centrum Wiskunde and Informatica, Amsterdam, The Netherlands
}

\section{A R T I C L E I N F O}

\section{Article history:}

Received 9 May 2017

Received in revised form 31 August 2017

Accepted 30 September 2017

Available online 9 October 2017

\section{Keywords:}

Stokes equations

Poroelastic equations

Interface conditions

Coupled system

Multigrid method

Vanka smoother

Uzawa smoother

Local Fourier analysis

\begin{abstract}
A B S T R A C T
The interaction between fluid flow and a deformable porous medium is a complicated multi-physics problem, which can be described by a coupled model based on the Stokes and poroelastic equations. A monolithic multigrid method together with either a coupled Vanka smoother or a decoupled Uzawa smoother is employed as an efficient numerical technique for the linear discrete system obtained by finite volumes on staggered grids. A specialty in our modeling approach is that at the interface of the fluid and poroelastic medium, two unknowns from the different subsystems are defined at the same grid point. We propose a special discretization at and near the points on the interface, which combines the approximation of the governing equations and the considered interface conditions. In the decoupled Uzawa smoother, Local Fourier Analysis (LFA) helps us to select optimal values of the relaxation parameter appearing. To implement the monolithic multigrid method, grid partitioning is used to deal with the interface updates when communication is required between two subdomains. Numerical experiments show that the proposed numerical method has an excellent convergence rate. The efficiency and robustness of the method are confirmed in numerical experiments with typically small realistic values of the physical coefficients.
\end{abstract}

(c) 2017 Elsevier Inc. All rights reserved.

\section{Introduction}

The interaction between a free fluid and a poroelastic material is attracting researchers' attention because of the wide range of applications. For example, in geomechanics, the interaction can be found in reservoir engineering, ground water flow modeling and oil extraction. Another example, in biology, is the behavior of blood flow through the main vessels. All the examples are governed by the coupling of solid and fluid mechanics. In order to detect or control the flow and the deformation of the porous medium, it is of great importance to understand the mechanism of the coupled dynamical process.

To model the incompressible and Newtonian free flow, the Stokes equations are considered here. The behavior of the deformable fluid-saturated porous medium is described by the well-developed Biot's model [1,2], in which the fluid motion and solid deformation are taken into account. In Biot's model, the fluid in the pores is modeled by the Darcy equation, and the deformation by elasticity, resulting in the poroelastic equations. It is a challenge to couple the Stokes and poroelasticity

\footnotetext{
* Corresponding author.

E-mail address: p.luo@tudelft.nl (P. Luo).
} 
systems properly at the interface. For this purpose, the conservation of mass and momentum, and a balance of normal stress and tangential stress equilibria are imposed at the interface. To our knowledge, the coupled model has not been widely studied yet, probably due to the complex coupling at the interface. In [3], Showalter models the interaction between the Stokes and the Biot's poroelasticity equations, and shows the model to be a mathematical well-posed problem which is amenable to analysis and computation.

From the computational viewpoint, the finite volume method on a staggered grid is taken into consideration as the discrete scheme for the coupled Stokes-poroelasticity system. A challenge is to deal with the discretization at the interface, since two unknowns belonging to different subsystems are placed at the same location. In this special discrete system, the governing equations of both free fluid and deformable porous medium, and their complex interaction are all included, showing a strong coupling between the two subsystems. Based on the discretization, a linear system of saddle point structure [4] is obtained.

Basically, there are two ways to solve a coupled multi-physics system. First of all, a popular approach is the domain decomposition (DD) method. In [5], a DD approach using the SIMPLE-algorithm [6] on a cell-centered grid for the Stokes equations and a multigrid solver on a staggered grid for the Biot equations is employed. The authors in [7] adopt an extended DD method for the fluid-poroelastic structure interaction (FPSI) problem without considering the fluid motion in the porous medium. It is a modular approach which only requires interface data transfer between the two existing fluid and structure codes, without any modification of the sources. In [8], partitioning strategies based on a Nitsche's coupling approach are developed for the coupled Stokes-Biot system, while in [9] a loosely coupled finite element solver by considering a Lie operator splitting scheme is proposed for the coupled fluid-structure interaction.

A second approach is to consider monolithic methods. These are algorithms that are developed for solving the fully coupled system at once. As a highly efficient solver for a saddle point system, the monolithic multigrid method is chosen in this paper. Methods of this type have been successfully applied for the system of Stokes equations [10,11], and also for poroelasticity equations [12-14]. In any multigrid method, the smoothing strategy is an important component which impacts the convergence crucially. Basically, multigrid smoothers for systems of partial differential equations can be classified into two major categories: coupled and decoupled smoothers [15,16]. In this paper, both types of smoothers are considered for the Stokes-poroelasticity system. With respect to the coupled smoothers, the Vanka smoother [10], or box relaxation, in which the unknowns appearing in the discrete divergence operator are relaxed simultaneously, is popular and robust. Decoupled smoothers, or equation-wise relaxations, are often chosen because of their convenient implementation and their low computational cost, especially when solving problems on stretched grids. Here, we also consider a decoupled smoother called the Uzawa smoother for the coupled system. In [12], [11] and [17], the Uzawa smoother was successfully applied for solving poroelastic equations and Stokes problems, respectively. The convergence behavior of multigrid relies on the values of relaxation parameters that, when using the Uzawa smoother, are different for each subsystem and grid of the multigrid hierarchy. To choose optimal values of the parameters, local Fourier analysis (LFA), introduced by Brandt [18], is applied.

Finally, from the implementation point of view, we consider a monolithic multigrid based on grid partitioning [16], where communication between neighboring subdomains is needed on each multigrid level.

The paper is organized as follows. First of all, we introduce the governing equations of free fluid and deformable porous medium, as well as the interface conditions in Section 2. The discretization of the coupled Stokes-poroelasticity system is shown in Section 3. In particular, we present the details about how we deal with the interface. The monolithic multigrid method, together with the coupled and decoupled smoothers, are shown in Section 4. After that, several numerical experiments are given to confirm the efficiency and robustness of our proposed method. Conclusions are drawn in Section 6. Some finer details about the discretizations, the derivation of the optimal values for the relaxation parameter and the communication within grid partitioning are found in the appendices.

\section{Problem formulation}

The Stokes-poroelasticity problem is considered on a bounded domain $\Omega \subset \mathbb{R}^{2}$. It is assumed that $\Omega$ is subdivided into two disjoint subdomains $\Omega^{f}$ and $\Omega^{p}$, separated by a common interface $\Gamma=\partial \Omega^{f} \cap \partial \Omega^{p}$. Subregions $\Omega^{f}$ and $\Omega^{p}$ represent the free flow region and poroelastic medium, respectively. A model geometry of the problem is shown in Fig. 1, where we also display $\mathbf{n}^{f}$ and $\mathbf{n}^{p}$, denoting the unit outward normal vectors on $\partial \Omega^{f}$ and $\partial \Omega^{p}$, respectively. At the interface $\Gamma$, we have $\mathbf{n}^{f}=-\mathbf{n}^{p}$.

The description of the free flow and poroelasticity models on the different subdomains, including the boundary conditions for the outer boundaries, are presented in Sections 2.1 and 2.2. The internal interface conditions governing the interactions between the fluid and the porous medium are given in Section 2.3.

\subsection{Stokes flow description}

The 2D free flow subproblem is modeled by the Stokes equations for a viscous, incompressible, Newtonian fluid. The motion of the Stokes flow in the region $\Omega^{f}$ is described by 


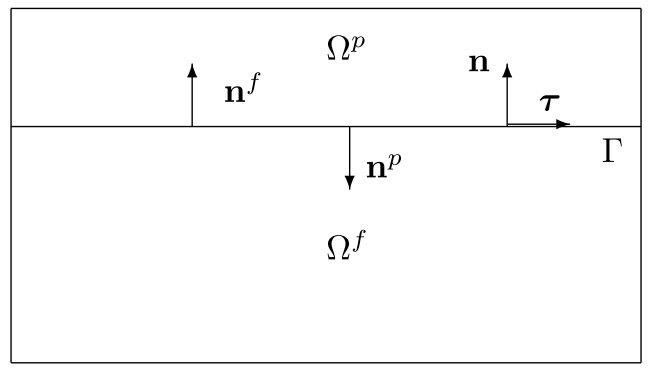

$\Omega$

Fig. 1. Example of a model geometry of the Stokes-poroelasticity problem. Subdivision of the domain $\Omega$ into a free flow subregion $\Omega^{f}$ and a porous medium subdomain $\Omega^{p}$, separated by an internal interface $\Gamma$.

$$
\begin{aligned}
\rho \frac{\partial \mathbf{u}^{f}}{\partial t}-\nabla \cdot \boldsymbol{\sigma}^{f}=\mathbf{f}^{f} & \text { in } \Omega^{f}, 0<t \leq T, \\
\nabla \cdot \mathbf{u}^{f}=0 & \text { in } \Omega^{f}, 0<t \leq T,
\end{aligned}
$$

where $\rho$ is the fluid density, $\mathbf{u}^{f}=\left(u^{f}, v^{f}\right)$ is the fluid velocity, $\mathbf{f}^{f}=\left(f_{1}^{f}, f_{2}^{f}\right)$ represents a prescribed force, and the fluid stress tensor $\boldsymbol{\sigma}^{f}$ is given by $\boldsymbol{\sigma}^{f}=-p^{f} \mathbf{I}+2 v \mathbf{D}\left(\mathbf{u}^{f}\right)$, with $p^{f}$ denoting the fluid pressure, $v$ representing the fluid viscosity and where $\mathbf{D}\left(\mathbf{u}^{f}\right)=\left(\nabla \mathbf{u}^{f}+\left(\nabla \mathbf{u}^{f}\right)^{T}\right) / 2$ is the strain tensor. The first formula in (1) represents the momentum equations and the second one is the continuity equation. We assume that the boundary $\partial \Omega^{f} \backslash \Gamma$ is the union of two disjoint subsets $\Gamma_{D}^{f}$ and $\Gamma_{N}^{f}$, where Dirichlet and Neumann boundary conditions are imposed, respectively. More concretely, we consider the following boundary conditions

$$
\begin{aligned}
\mathbf{u}^{f}=\mathbf{g}_{D}^{f} & \text { on } \Gamma_{D}^{f}, 0<t \leq T, \\
\boldsymbol{\sigma}^{f} \cdot \mathbf{n}^{f}=\mathbf{g}_{N}^{f} & \text { on } \Gamma_{N}^{f}, 0<t \leq T .
\end{aligned}
$$

\subsection{Poroelastic flow description}

We consider the 2D quasi-static Biot's model to represent the behavior of a deformable fluid-saturated porous medium. The porous medium is assumed to be linearly elastic, homogeneous and isotropic, and the fluid is supposed to be incompressible and viscous. The governing equations in subdomain $\Omega^{p}$ are given by

$$
\begin{array}{r}
-\nabla \cdot \boldsymbol{\sigma}^{p}=\mathbf{f}^{p} \text { in } \Omega^{p}, 0<t \leq T, \\
\frac{\partial}{\partial t}\left(\nabla \cdot \mathbf{u}^{p}\right)+\nabla \cdot \mathbf{q}^{p}=f^{p} \text { in } \Omega^{p}, 0<t \leq T, \\
\mathbf{q}^{p}=-K \nabla p^{p} \text { in } \Omega^{p}, 0<t \leq T .
\end{array}
$$

The stress tensor of the poroelastic medium is $\sigma^{p}=\sigma^{e}-p^{p} \mathbf{I}$, where $\sigma^{e}$ denotes the effective stress tensor and $p^{p}$ is the fluid pressure. The right-hand side $\mathbf{f}^{p}=\left(f_{1}^{p}, f_{2}^{p}\right)$ represents the external body forces, whereas the source term $f^{p}$ corresponds to a forced fluid extraction or injection process. Since the porous skeleton is assumed to be deformable, the effective stress tensor $\boldsymbol{\sigma}^{e}$ is characterized by the displacement $\mathbf{u}^{p}=\left(u^{p}, v^{p}\right)$, i.e. $\boldsymbol{\sigma}^{e}\left(\mathbf{u}^{p}\right)=2 \mu \mathbf{D}\left(\mathbf{u}^{p}\right)+\lambda \operatorname{tr}\left(\mathbf{D}\left(\mathbf{u}^{p}\right)\right) \mathbf{I}$, where $\lambda$ and $\mu$ denote the Lamé coefficients for the solid framework and $\mathbf{D}\left(\mathbf{u}^{p}\right)=\left(\nabla \mathbf{u}^{p}+\left(\nabla \mathbf{u}^{p}\right)^{T}\right) / 2$. Parameter $K$ is the hydraulic conductivity, representing the properties of the porous medium and the fluid. The flux $\mathbf{q}^{p}$ is the relative velocity of the fluid within the porous matrix. For the poroelasticity subproblem, at the outer boundary, $\partial \Omega^{p} \backslash \Gamma$, we prescribe combinations of the following boundary conditions,

$$
\begin{aligned}
\mathbf{u}^{p} & =\mathbf{g}_{D}^{p}, 0<t \leq T, \\
p^{p} & =g_{D}^{p}, 0<t \leq T, \\
\boldsymbol{\sigma}^{p} \cdot \mathbf{n}^{p} & =\mathbf{g}_{N}^{p}, 0<t \leq T, \\
\mathbf{q}^{p} \cdot \mathbf{n}^{p} & =g_{N}^{p}, 0<t \leq T .
\end{aligned}
$$

\subsection{Interface conditions}

To solve the coupled system, proper internal interface conditions are needed to be set up at the interface $\Gamma$. Here we denote by $\mathbf{n}=\mathbf{n}^{f}=-\mathbf{n}^{p}$ the outward normal vector to the fluid domain and by $\boldsymbol{\tau}$ the tangential unit vector on the interface $\Gamma$, which is obtained by rotating the normal vector in the counter-clockwise direction by $90^{\circ}$. It is natural to consider the continuity of velocities and stresses at the interface of the fluid and the poroelastic medium. 
- For mass conservation, the continuity of normal fluid flux across the interface is required,

$$
\left(\mathbf{u}^{f}-\frac{\partial \mathbf{u}^{p}}{\partial t}\right) \cdot \mathbf{n}=\mathbf{q}^{p} \cdot \mathbf{n},
$$

where $\frac{\partial \mathbf{u}^{p}}{\partial t}$ is the velocity of the skeleton, and the flux $\mathbf{q}^{p}$ denotes the filtration velocity.

- Regarding the exchange of stresses, the normal components of the stress in the fluid phase should be in balance

$$
\mathbf{n} \cdot \boldsymbol{\sigma}^{f} \mathbf{n}=-p^{p} .
$$

- The conservation of momentum prescribes the balance of contact forces, i.e. the stress of the porous medium is balanced by the stress of the fluid, that is,

$$
\mathbf{n} \cdot \boldsymbol{\sigma}^{f} \mathbf{n}-\mathbf{n} \cdot \boldsymbol{\sigma}^{p} \mathbf{n}=0
$$

and

$$
\boldsymbol{\tau} \cdot \boldsymbol{\sigma}^{f} \mathbf{n}-\boldsymbol{\tau} \cdot \boldsymbol{\sigma}^{p} \mathbf{n}=0
$$

- A no-slip interface boundary condition is considered,

$$
\mathbf{u}^{f} \cdot \boldsymbol{\tau}=\frac{\partial \mathbf{u}^{p}}{\partial t} \cdot \boldsymbol{\tau},
$$

for the cases in which there is no tangential flow.

Remark. Besides the no-slip interface conditions, also the Beavers-Joseph-Saffman interface condition

$$
-\boldsymbol{\tau} \cdot \boldsymbol{\sigma}^{f} \mathbf{n}=\beta\left(\mathbf{u}^{f}-\frac{\partial \mathbf{u}^{p}}{\partial t}\right) \cdot \boldsymbol{\tau},
$$

is sometimes used in problems where porous media and free flow domains are connected. The slip rate coefficient $\beta$ quantifies the resistance of the porous medium to the flow of the fluid in tangential direction. The parameter that needs to be determined in this interface condition is non-trivial and often connected to real experiments. Therefore, we do not consider the Beavers-Joseph-Saffman condition here for the Stokes-poroelasticity multi-physics experiments. We would like to note, however, that the strategy proposed in this work can be also applied if the Beavers-Joseph-Saffman interface condition would be employed.

\section{Discretization}

We consider the finite volume method on a staggered grid [19] as the spatial discretization scheme for the coupled Stokes-poroelasticity problem. An advantage of this discretization is that spurious oscillations do not appear in the numerical solution [20,21], and mass conservation is ensured. The implicit Euler scheme is taken for the time discretization.

The computational domain is partitioned into square blocks of size $h \times h$, so that the grid is conforming at the interface $\Gamma$. For notational convenience, we choose equal-sized blocks but the description for the more general case would be straightforward. Different control volumes are defined depending on the variable considered. In Fig. 2, we represent in different colors the control volumes associated with the equations collocated with the primary variables $u, v$ and $p .{ }^{1}$

Our aim is to obtain a special discrete equation for the unknowns at and near the internal interface $\Gamma$. Details for the discretization of the interior points of the poroelastic and Stokes equations are shown in Appendix A. Due to the staggered arrangement of the unknowns, the only variables at the interface are the vertical components of each system. Note that at the interface, two unknowns $v^{p}$ and $v^{f}$ share the same location, so we have two different governing equations at one point, see Fig. 3.

Remark. For the case in which the interface does not match with the grid points, we refer to the modeling as done in [5,22]. In [5], a cell-centered grid in the fluid region and a staggered grid in the porous domain are used for the coupled system. To deal with the discretization of the interface conditions, the authors introduce some specific grid points at the interface, where the information between the two subdomains is exchanged. The coupling with these additional points is done by means of a matching function, which can, for example, be a bilinear interpolation. Alternatively, the Mortar finite element method [22] is a suitable technique for interface problems when considering the coupling of different discretization schemes or non-matching meshes.

\footnotetext{
1 In this figure, the superscript, either $p$ or $f$, is omitted, as we have the same arrangement of unknowns for both sub-problems.
} 


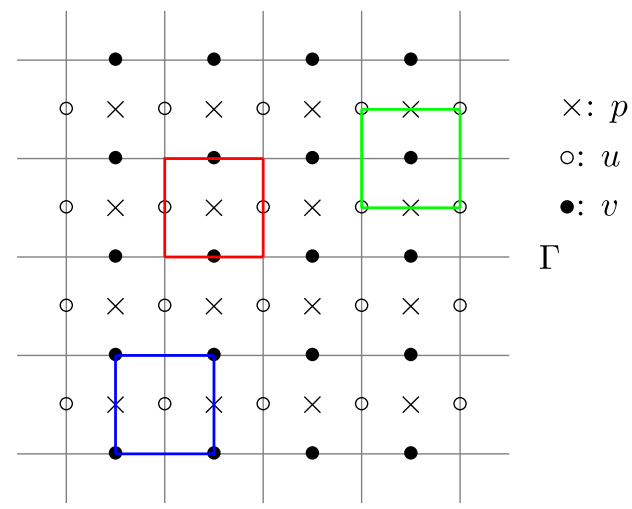

Fig. 2. Staggered grid location of unknowns for the coupled model, and corresponding control volumes. (For interpretation of the references to color in this figure, the reader is referred to the web version of this article.)

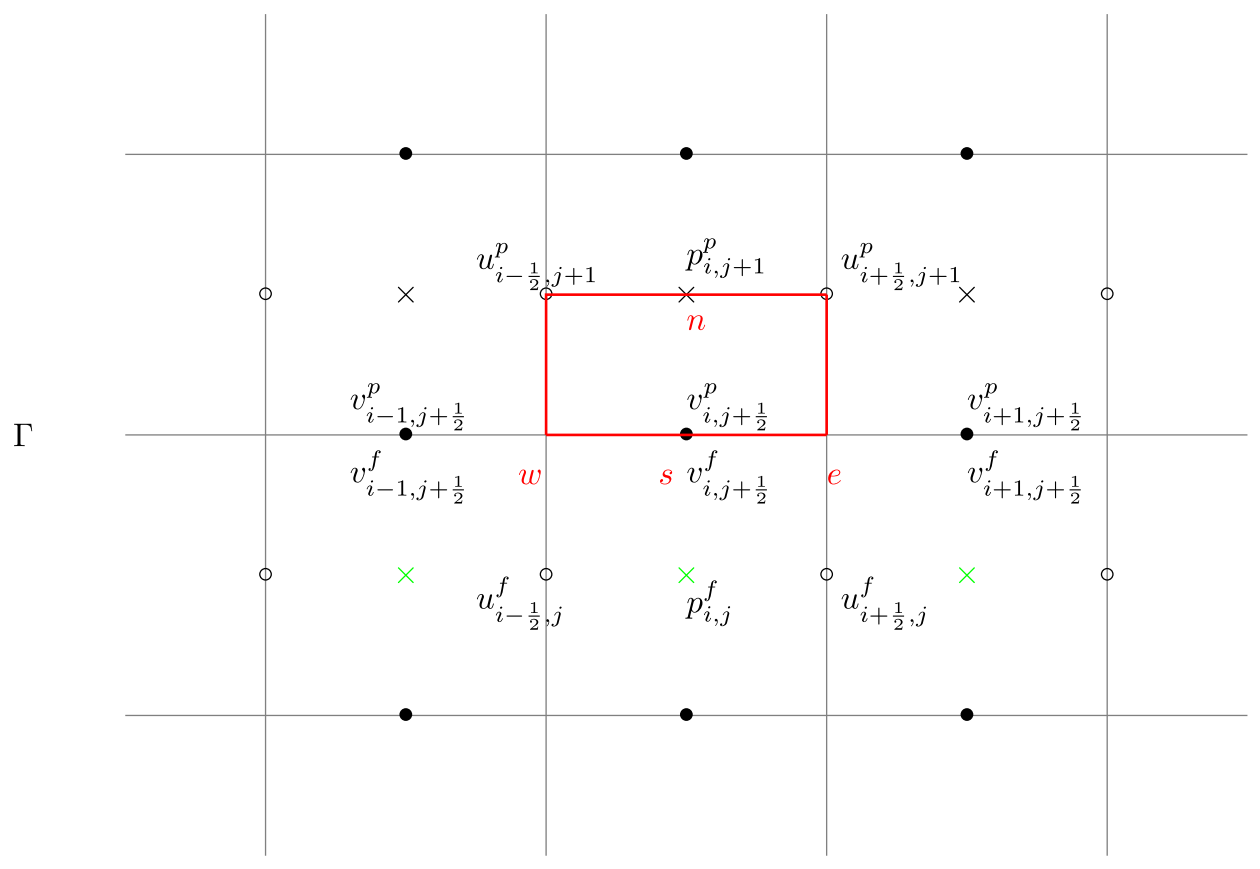

Fig. 3. Staggered grid location of the unknowns and the control volume of $v^{p}$ at the interface. (For interpretation of the references to color in this figure, the reader is referred to the web version of this article.)

\subsection{Discretization for $v^{p}$ and $v^{f}$ at the interface}

First of all, we describe the discretization for the poroelastic unknown $v^{p}$ at the interface. Note that in the following discrete formula, the superscript $m$ represents the values at current time, while $m-1$ denotes the solutions from a previous time step. For simplicity, we consider uniform meshes with time step $\tau$ and grid size $h$ in both directions on each subdomain. The $y$-momentum equation of the poroelastic system is integrated over a half volume, as displayed in red color in Fig. 3, giving rise to the following equation

$$
-\left(\frac{\left(\sigma_{x y}^{p, m}\right)_{e}-\left(\sigma_{x y}^{p, m}\right)_{w}}{h}+\frac{\left(\sigma_{y y}^{p, m}\right)_{n}-\left(\sigma_{y y}^{p, m}\right)_{s}}{h / 2}\right)=\left(f_{2}^{p, m}\right)_{s},
$$

where, as can be seen in Fig. 3, $e$ and $w$ denote locations at the interface, and $n$ and $s$ denote the location of $p_{i, j+1}^{p}$ and $v_{i, j+\frac{1}{2}}^{p} / v_{i, j+\frac{1}{2}}^{f}$, respectively. In the discrete formula, for example, we denote the approximation of $v^{p}$ in the grid point $\left(i, j+\frac{1}{2}\right)$ at the interface as $v_{i, j+\frac{1}{2}}^{p}$. Similar notations are used for other variables. $\left(\sigma_{y y}^{p, m}\right)_{n}$ is easily approximated by the existing variables as follows, 


$$
\left(\sigma_{y y}^{p, m}\right)_{n}=\frac{\lambda}{h}\left(u_{i+\frac{1}{2}, j+1}^{p, m}-u_{i-\frac{1}{2}, j+1}^{p, m}\right)+\frac{\lambda+2 \mu}{h}\left(v_{i, j+\frac{3}{2}}^{p, m}-v_{i, j+\frac{1}{2}}^{p, m}\right)-p_{i, j+1}^{p, m} .
$$

For the remaining components of the stress tensor, the interface conditions are required. By applying the interface conditions (6) and (7), $\left(\sigma_{y y}^{p, m}\right)_{s}$ is obtained as,

$$
\left(\sigma_{y y}^{p, m}\right)_{s}=\left(\sigma_{y y}^{f, m}\right)_{s}=-p_{s}^{p, m}
$$

The stress tensors $\left(\sigma_{x y}^{p, m}\right)_{e}$ and $\left(\sigma_{x y}^{p, m}\right)_{w}$ are related to the horizontal displacements $u_{e}^{p, m}$ and $u_{w}^{p, m}$ at the interface as,

$$
\begin{aligned}
& \left(\sigma_{x y}^{p, m}\right)_{e}=\mu\left(\frac{u_{i+\frac{1}{2}, j+1}^{p, m}-u_{e}^{p, m}}{h / 2}+\frac{v_{i+1, j+\frac{1}{2}}^{p, m}-v_{i, j+\frac{1}{2}}^{p, m}}{h}\right), \\
& \left(\sigma_{x y}^{p, m}\right)_{w}=\mu\left(\frac{u_{i-\frac{1}{2}, j+1}^{p, m}-u_{w}^{p, m}}{h / 2}+\frac{v_{i, j+\frac{1}{2}}^{p, m}-v_{i-1, j+\frac{1}{2}}^{p, m}}{h}\right) .
\end{aligned}
$$

Substituting (12)-(15) into (11) gives rise to the following discrete equation for $v_{i, j+\frac{1}{2}}^{p, m}$,

$$
\begin{aligned}
& -\frac{2(\lambda+\mu)}{h^{2}}\left(u_{i+\frac{1}{2}, j+1}^{p, m}-u_{i-\frac{1}{2}, j+1}^{p, m}\right)+\frac{2 \mu}{h^{2}}\left(u_{e}^{p, m}-u_{w}^{p, m}\right)-\frac{\mu}{h^{2}}\left(v_{i+1, j+\frac{1}{2}}^{p, m}+v_{i-1, j+\frac{1}{2}}^{p, m}\right) \\
& -\frac{2(\lambda+2 \mu)}{h^{2}} v_{i, j+\frac{3}{2}}^{p, m}+\left(\frac{2 \mu}{h^{2}}+\frac{2(\lambda+2 \mu)}{h^{2}}\right) v_{i, j+\frac{1}{2}}^{p, m}+\frac{2}{h}\left(p_{i, j+1}^{p, m}-p_{s}^{p, m}\right)=\left(f_{2}^{p, m}\right)_{s} .
\end{aligned}
$$

Since pressure $p_{s}^{p, m}$ and the two displacements $u_{e}^{p, m}$ and $u_{w}^{p, m}$ are not known at the interface, we approximate them with the help of the interface conditions. From the interface condition (5), the approximation of $p_{s}^{p, m}$ reads

$$
p_{s}^{p, m}=p_{i, j+1}^{p, m}+\frac{h}{2 K} v_{i, j+\frac{1}{2}}^{f, m}-\frac{h}{2 K \tau}\left(v_{i, j+\frac{1}{2}}^{p, m}-v_{i, j+\frac{1}{2}}^{p, m-1}\right) .
$$

To approximate the horizontal displacement, we need to use the conservation of momentum (8) and the no-slip interface condition (9). Thus, by applying (8) and (9) at the location denoted by $e$ in Fig. 3, we obtain the expressions of $u_{e}^{p, m}$ and $u_{e}^{f, m}$ as

$$
u_{e}^{p, m}=\mu \tau \xi\left(2 u_{i+\frac{1}{2}, j+1}^{p, m}+v_{i+1, j+\frac{1}{2}}^{p, m}-v_{i, j+\frac{1}{2}}^{p, m}\right)-v \tau \xi\left(-2 u_{i+\frac{1}{2}, j}^{f, m}+v_{i+1, j+\frac{1}{2}}^{f, m}-v_{i, j+\frac{1}{2}}^{f, m}\right)+2 v \xi u_{e}^{p, m-1},
$$

and

$$
u_{e}^{f, m}=\mu \xi\left(2 u_{i+\frac{1}{2}, j+1}^{p, m}+v_{i+1, j+\frac{1}{2}}^{p, m}-v_{i, j+\frac{1}{2}}^{p, m}\right)-v \xi\left(-2 u_{i+\frac{1}{2}, j}^{f, m}+v_{i+1, j+\frac{1}{2}}^{f, m}-v_{i, j+\frac{1}{2}}^{f, m}\right)-2 \mu \xi u_{e}^{p, m-1}
$$

where $\xi=\frac{1}{2 v+2 \mu \tau}$. The approximation of the horizontal velocity at the location $w$ can be calculated in a similar way. Then, by applying (17), (18) and the approximation of $u_{w}^{p, m}$ to (16), we have the discrete formula for the interface unknown $v_{i, j+\frac{1}{2}}^{p, m}$. This discretization does not only include the poroelastic unknowns but also the unknowns of the Stokes system. When implementing the discretization for one subsystem, the information from the other subsystem is required. Strong coupling is thus guaranteed through the interface conditions.

For the discretization of the second unknown $v^{f}$ at the interface, we obtain (20) by integrating the corresponding equation over a half control volume

$$
-\left(\frac{\left(\sigma_{x y}^{f, m}\right)_{e}-\left(\sigma_{x y}^{f, m}\right)_{w}}{h}+\frac{\left(\sigma_{y y}^{f, m}\right)_{n}-\left(\sigma_{y y}^{f, m}\right)_{s}}{h / 2}\right)+\frac{\rho}{\tau}\left(v_{i, j+\frac{1}{2}}^{f, m}-v_{i, j+\frac{1}{2}}^{f, m-1}\right)=\left(f_{2}^{f, m}\right)_{n} .
$$

Following the same technique to approximate the components of the stress tensor as above, we again obtain a formulation where three variables $u_{e}^{f, m}, u_{w}^{f, m}$ and $p_{n}^{p, m}$ are not known at the interface. The variables $u_{e}^{f, m}$ and $u_{w}^{f, m}$ can be calculated from the interface conditions (8) and (9). By applying these expressions to (20), the unknowns for both subproblems are included in the coupling between the two systems.

\subsection{Discretization for $u^{p}$ and $u^{f}$ near the interface}

The discretizations for the unknowns near the interface are also special and of great importance. Considering the control volume in Fig. 4 in red, the governing equation for $u_{i+\frac{1}{2}, j+1}^{p}$ is equation (A.1), which is shown in Appendix A. The related 


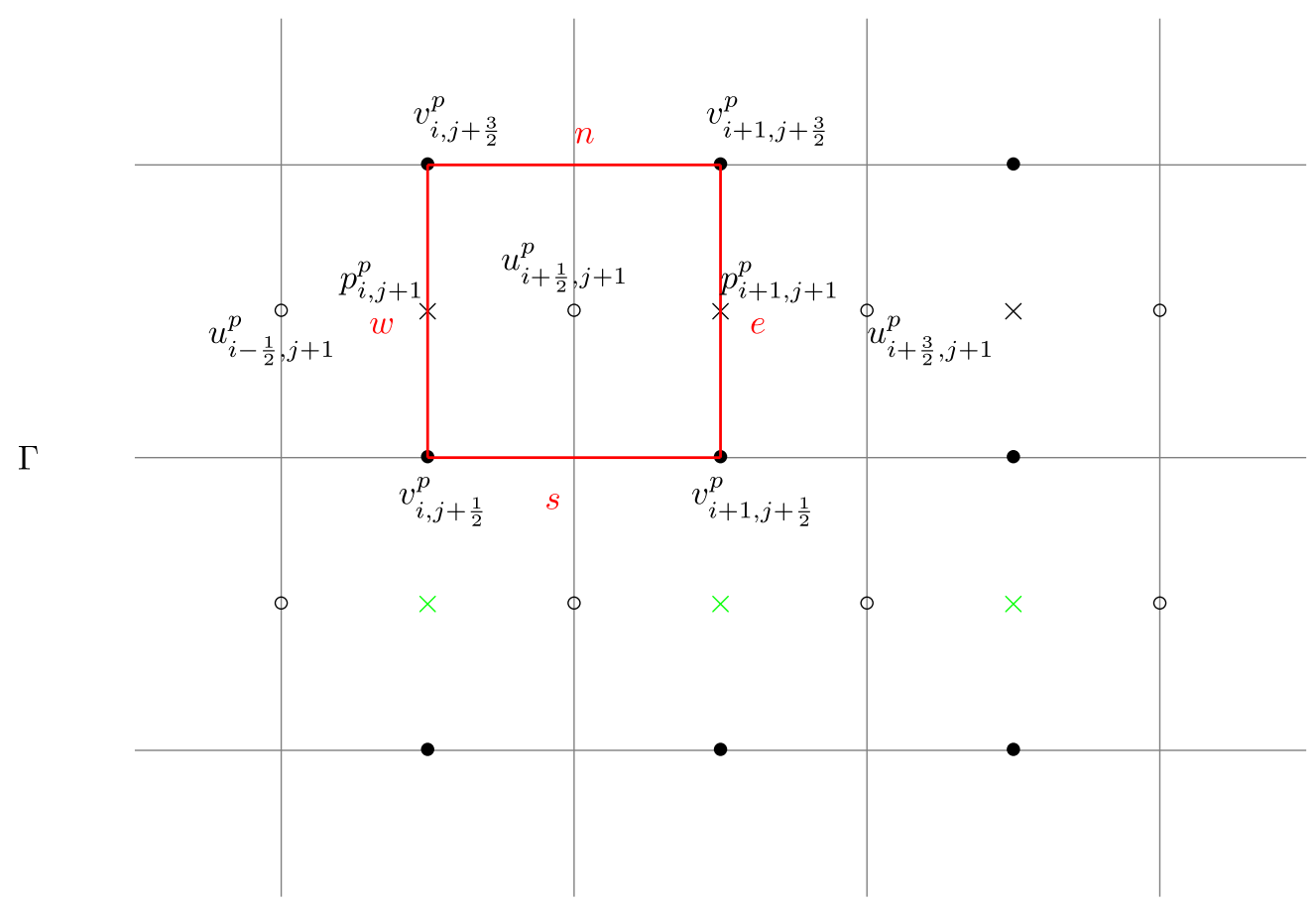

Fig. 4. Staggered grid location of the unknowns and the control volume of $u^{p}$ near the interface. (For interpretation of the references to color in this figure, the reader is referred to the web version of this article.)

stress components $\left(\sigma_{x x}^{p, m}\right)_{e},\left(\sigma_{x x}^{p, m}\right)_{w}$ and $\left(\sigma_{x y}^{p, m}\right)_{n}$ are similar to equations in (A.2)-(A.4). However, component $\left(\sigma_{x y}^{p, m}\right)_{s}$ is now written as

$$
\left(\sigma_{x y}^{p, m}\right)_{s}=\mu\left(\frac{u_{i+\frac{1}{2}, j+1}^{p, m}-u_{s}^{p, m}}{h / 2}+\frac{v_{i+1, j+\frac{1}{2}}^{p, m}-v_{i, j+\frac{1}{2}}^{p, m}}{h}\right),
$$

where the horizontal displacement $u_{s}^{p, m}$ at the interface is needed. A similar expression for $u_{s}^{p, m}$ can be obtained by using two interface conditions. Since $u_{s}^{p, m}$ includes the Stokes unknowns, the discrete equation for $u_{i+\frac{1}{2}, j+1}^{p, m}$ is also coupled with the free fluid system.

The discretization of $u_{i+\frac{1}{2}, j}^{f}$ can be obtained similarly.

\subsection{Discretization for $p^{p}$ and $p^{f}$ near the interface}

Following the notation in Fig. 5, the discretization for $p_{i, j+1}^{p}$ is given by

$$
\begin{aligned}
\frac{1}{h \tau}\left(u_{i+\frac{1}{2}, j+1}^{p, m}-u_{i-\frac{1}{2}, j+1}^{p, m}+v_{i, j+\frac{3}{2}}^{p, m}-\right. & \left.v_{i, j+\frac{1}{2}}^{p, m}\right)-\frac{K}{h^{2}}\left(p_{i+1, j+1}^{p, m}+p_{i-1, j+1}^{p, m}+p_{i, j+2}^{p, m}-5 p_{i, j+1}^{p, m}+2 p_{s}^{p, m}\right) \\
& =\left(f^{p, m}\right)_{i, j+1}+\frac{1}{h \tau}\left(u_{i+\frac{1}{2}, j+1}^{p, m-1}-u_{i-\frac{1}{2}, j+1}^{p, m-1}+v_{i, j+\frac{3}{2}}^{p, m-1}-v_{i, j+\frac{1}{2}}^{p, m-1}\right) .
\end{aligned}
$$

From the interface condition (5), $p_{s}^{p, m}$ can be approximated using the same strategy as in (17). For the Stokes pressure near the interface, the discrete equation remains the same as in (A.12) in Appendix A.

\subsection{Saddle point structure}

For the individual poroelastic and Stokes systems, the discretization described in the previous subsections leads to a saddle point linear system at each time step, of the form,

$$
\left(\begin{array}{cc}
A & B^{T} \\
B & -C
\end{array}\right)\left(\begin{array}{l}
\mathbf{u} \\
p
\end{array}\right)=\left(\begin{array}{l}
\mathbf{g} \\
f
\end{array}\right) .
$$




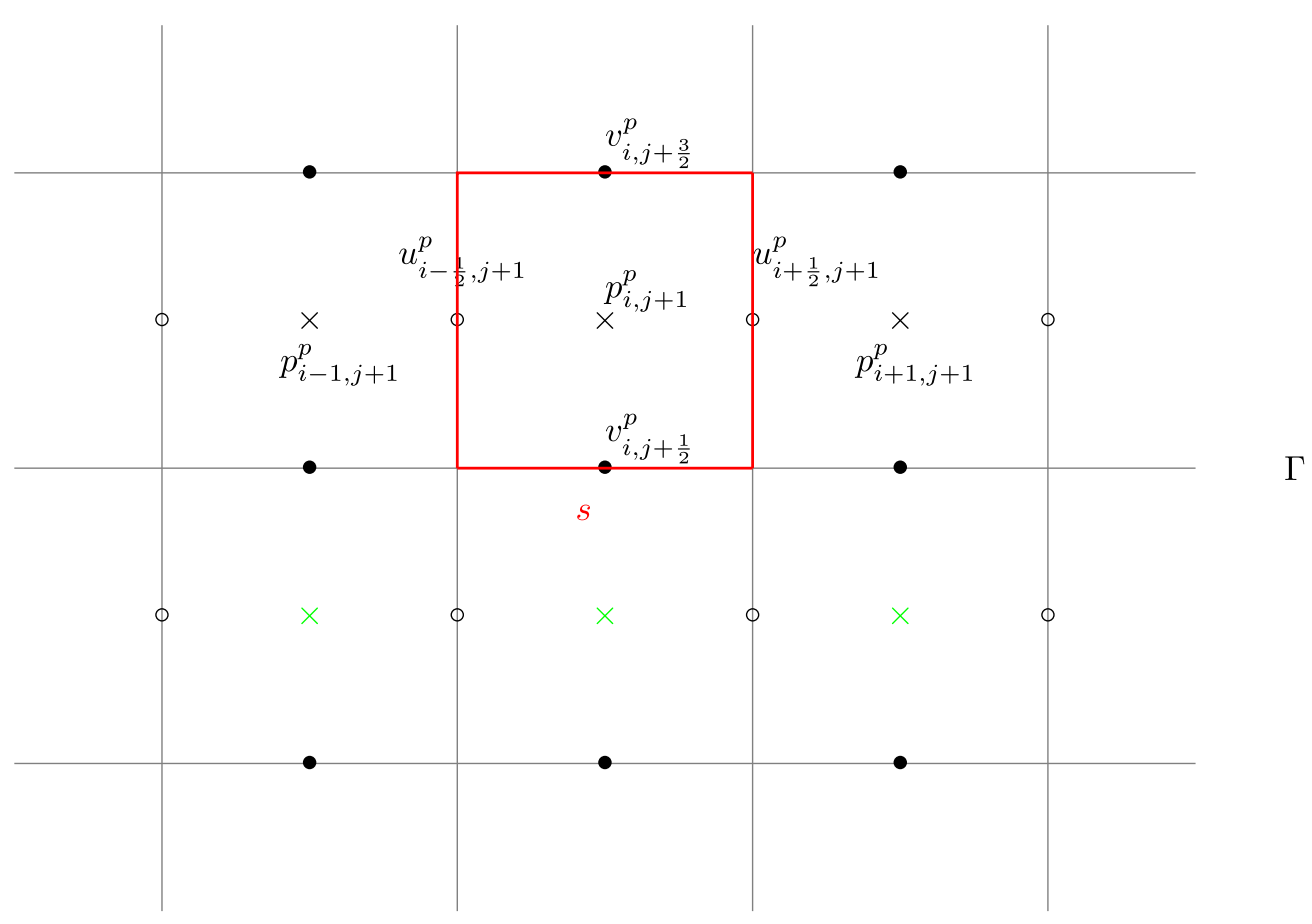

Fig. 5. Staggered grid location of the unknowns and the control volume of $p^{p}$ near the interface. (For interpretation of the references to color in this figure, the reader is referred to the web version of this article.)

For both subproblems $B^{T}$ and $B$ represent the discrete gradient and the negative discrete divergence operators, respectively. For the poroelastic system, $A$ is the discrete representation of the elastic operator $-\mu \Delta-\nabla(\lambda+\mu) \nabla \cdot$, and $C$ corresponds to the diffusion operator $-\tau \nabla \cdot(K \nabla p)$, whereas for the Stokes system, $A$ represents the discretization of operator $\frac{\rho}{\tau} I-v \Delta$ and $C$ is a zero block. Although the term in $C$ is nonzero for the poroelasticity subsystem, it can become arbitrarily small because parameter $K$ in the diffusion operator can be very small.

Analogously to the individual systems, the coupled system is also governed by the saddle point structure by rearranging the vector of unknowns to order first the velocities and displacements and thereafter the pressure unknowns. Thus the following linear system is obtained,

$$
\left(\begin{array}{cccc}
A^{f} & R^{T} & \left(B^{f}\right)^{T} & \left(R^{\prime}\right)^{T} \\
R & A^{p} & 0 & \left(B^{p}\right)^{T} \\
B^{f} & 0 & 0 & 0 \\
R^{\prime} & B^{p} & 0 & -C^{p}
\end{array}\right)\left(\begin{array}{c}
\mathbf{u}^{f} \\
\mathbf{u}^{p} \\
p^{f} \\
p^{p}
\end{array}\right)=\left(\begin{array}{c}
\mathbf{f}^{f} \\
\mathbf{f}^{p} \\
0 \\
f^{p}
\end{array}\right) .
$$

Obviously, (24) has the same structure as (23) by denoting

$$
A=\left(\begin{array}{cc}
A^{f} & R^{T} \\
R & A^{p}
\end{array}\right), B=\left(\begin{array}{cc}
B^{f} & 0 \\
R^{\prime} & B^{p}
\end{array}\right),-C=\left(\begin{array}{cc}
0 & 0 \\
0 & -C^{p}
\end{array}\right),
$$

where $R$ and $R^{\prime}$ are matrices containing the coupling for the unknowns at and near the interface. For each equation, most of the elements in $R$ and $R^{\prime}$ are zero, whereas the nonzero elements correspond to the terms not belonging to the current subsystem, but appearing in the special interface discretizations. In particular, the nonzero elements are on the diagonal of $R^{\prime}$. In the discretization of the vertical component of $\mathbf{u}^{f}$ at the interface, the nonzero elements in $R^{\prime}$ represent the appearance of poroelastic pressure $p^{p}$, while in the discrete equation of $p^{p}$, the vertical component of velocity $\mathbf{u}^{f}$ is present. $R$ has more nonzero elements than $R^{\prime}$, since the discretizations for $\mathbf{u}^{f}$ and $\mathbf{u}^{p}$ are more involved. For example, the Stokes velocity at three grid points is needed when discretizing the vertical component of $\mathbf{u}^{p}$ at the interface, whereas in the discretization of the horizontal component of $\mathbf{u}^{p}$ near the interface, only two Stokes velocities are necessary.

\section{Fast solvers based on multigrid}

Our aim is to design a monolithic geometric multigrid method for the coupled Stokes-poroelasticity problem. To obtain an efficient multigrid solver, it is necessary to carefully select the components of the algorithm. In this section, the smoothing operator, the restriction and prolongation operators and the coarse-grid discretization are all presented. 


\subsection{Smoother}

The smoother is an important multigrid component, which affects the performance of the method crucially. Basically, there are two types of robust smoothers developed for our problem, classified as coupled and decoupled smoothers.

\subsubsection{Coupled smoothers}

Vanka smoother Regarding coupled smoothers, the Vanka smoother, which is also called box relaxation, is considered here. The Vanka smoother was originally proposed for the incompressible Navier-Stokes equations [10] and can be straightforwardly applied to solving the Stokes-poroelasticity problem. For the staggered arrangement of variables, this approach is based on updating all equations of the system for each grid cell simultaneously. Particularly, five unknowns $\left(u_{i-\frac{1}{2}, j}, u_{i+\frac{1}{2}, j}\right.$, $v_{i, j+\frac{1}{2}}, v_{i, j-\frac{1}{2}}$ and $p_{i, j}$ ) as shown in Fig. A.16 (right) in Appendix A are relaxed at the same time. The relaxation is implemented in a block Gauss-Seidel fashion and for each cell in the grid, a $5 \times 5$-system is solved. For the poroelasticity system, a multigrid method based on the Vanka smoother has been applied in [14] showing a very robust performance.

\subsubsection{Decoupled smoother}

Uzawa smoother Regarding the decoupled smoothers, we focus on the so-called Uzawa smoother, which is an equationwise relaxation method. The Uzawa smoother was proposed for Stokes problems in fluid dynamics in [11], [17]. In [12], a multigrid method with an Uzawa smoother was successfully applied for solving poroelastic equations. Thus, it seems natural to assume that this relaxation is also suitable for the coupled Stokes-poroelasticity problem. The smoother is obtained by splitting the discrete operator as follows

$$
\left(\begin{array}{cc}
A & B^{T} \\
B & -C
\end{array}\right)=\left(\begin{array}{cc}
M_{A} & 0 \\
B & -\omega^{-1} I
\end{array}\right)-\left(\begin{array}{cc}
M_{A}-A & -B^{T} \\
0 & C-\omega^{-1} I
\end{array}\right),
$$

where $M_{A}$ is a typical smoother for $A$ and $\omega$ is some positive parameter. The use of $M_{A}$ makes the approach less costly because of the inexact solve for the velocities and displacements at each iteration.

Supposing an approximation of the solution to the system $(\mathbf{u}, p)^{T}$ is given, the relaxed approximation $(\widehat{\mathbf{u}}, \widehat{p})^{T}$ is calculated according to the decoupled Uzawa smoother, as

$$
\left(\begin{array}{cc}
M_{A} & 0 \\
B & -\omega^{-1} I
\end{array}\right)\left(\begin{array}{l}
\widehat{\mathbf{u}} \\
\widehat{p}
\end{array}\right)=\left(\begin{array}{cc}
M_{A}-A & -B^{T} \\
0 & C-\omega^{-1} I
\end{array}\right)\left(\begin{array}{l}
\mathbf{u} \\
p
\end{array}\right)+\left(\begin{array}{l}
\mathbf{g} \\
f
\end{array}\right) .
$$

Here $M_{A}$ represents the symmetric Gauss-Seidel iteration given by

$$
M_{A}=\left(D_{A}+L_{A}\right) D_{A}^{-1}\left(D_{A}+U_{A}\right),
$$

where $D_{A}, L_{A}$ and $U_{A}$ are, respectively, the diagonal, the strictly lower, and the strictly upper parts of $A$. There are two important properties of $M_{A}$ needed in the theoretical analysis. One is that $M_{A}$ is SPD, if $A$ is SPD. The other is that the associated largest eigenvalue satisfies (see, e.g., [23, Theorem 7.17])

$$
\lambda_{\max }\left(M_{A}^{-1} A\right) \leq 1 .
$$

With respect to the pressure, the Uzawa method is equivalent to a Richardson iteration applied to the Schur complement system. The relaxation parameter $\omega$ in (25), (26) is set to minimize the spectral radius of the corresponding iteration matrix, i.e.,

$$
\omega=\frac{2}{\lambda_{\max }+\lambda_{\min }},
$$

where $\lambda_{\max }$ and $\lambda_{\min }$ denote the largest and smallest eigenvalues on the high frequency components of the Schur complement, respectively (see [17]). To estimate the optimal relaxation parameter, local Fourier analysis is employed. Note that the estimated relaxation parameters from LFA are optimal for periodic boundary conditions. They are often close to optimal in the more general case. The convergence behavior of the Uzawa smoother crucially depends on the choice of an adequate value for the relaxation parameter.

Optimal relaxation parameter In [12] and [17], different analytic expressions of $\omega$ for the individual Stokes and poroelastic systems are obtained by a theoretical analysis that we can use directly for the coupled system as well. Local Fourier analysis is a powerful tool for the quantitative analysis of multigrid. It helps to predict the asymptotic convergence factor of the multigrid algorithm and provides a concrete formula for the optimal relaxation parameter. In [12], the optimal parameter for the poroelastic system is given by

$$
\omega^{p}=\frac{h^{2}(\lambda+2 \mu)}{5 K \tau(\lambda+2 \mu)+h^{2}},
$$


with the parameters $K, \lambda, \mu, h, \tau$ as previously introduced. Meanwhile, the optimal parameter for the Stokes system is obtained from [17] as follows

$$
\omega^{f}=v+\frac{\rho h^{2}}{8 \tau} .
$$

From (28) and (29), it can be seen that the relaxation parameters do not only depend on the model coefficients but also on the grid size and on time step $\tau$, thus $\omega^{p}$ and $\omega^{f}$ are different on each grid of the hierarchy in the multigrid method. Details about the derivation of the optimal values for the relaxation parameter can be found in Appendix B.

Remark. Besides the smoothers presented in this paper, other relaxations may be considered, for example, the distributive Gauss-Seidel smoother (DGS). DGS is a smoother which, after an operator transformation, results in a decoupled, equationwise relaxation method and it has been designed for discretizations on staggered grids. Distributive smoothing methods for incompressible flow problems or poroelasticity systems have been presented in [16,24] and [14]. However, for the coupled Stokes-poroelasticity system, DGS method is rather challenging. It is not clear how to define the operator transformation due to the involved coupling of the poroelasticity and Stokes unknowns at and near the interface.

\subsubsection{Communication}

The computational domain is divided into (at least) two different blocks corresponding to the Stokes and poroelasticity domains. The points at and near $\Gamma$ have special discretizations that contain the variables from the neighboring subdomain. Therefore, it is necessary for each subgrid to store not only the data from its own but also a copy of data of the neighboring subgrid in an overlap region. The size of this overlap region should however be one grid cell only. The communication between two individual subsystems is based on the grid partitioning technique. The details of the communication between two subgrids are shown in Appendix C.

In the monolithic multigrid method, we choose the grids so that $\Gamma$ is present on the complete grid hierarchy. The communication between the Stokes and poroelasticity problems is performed at each grid level instead of only at the finest grid. After the smoothing process on each grid level, the values of unknowns in the overlap region are updated by communication.

Regarding the Uzawa smoother, the communication strategy is essentially the same as for the Vanka smoother. Here we wish to precisely describe the relaxation order of the unknowns in the Uzawa smoother which is different from that for the Vanka smoother. In the Uzawa smoothing process, all velocity and displacement unknowns are updated before all pressure unknowns are relaxed. Different relaxation parameters $\omega^{f / p}$ for the Richardson iteration for the Schur complement are chosen when updating the pressure unknowns in the Stokes and poroelastic domains. In a full smoothing iteration, we thus first relax the velocity $\mathbf{u}^{f}$ in $\Omega^{f}$, then the updates are transferred to the overlap region in $\Omega^{p}$. With these values, we update the displacement unknowns $\mathbf{u}^{p}$, and transfer them to the overlap region in $\Omega^{f}$. After that, we return to $\Omega^{f}$ to update the pressure $p^{f}$ by using relaxation parameter $\omega^{f}$ in (29), then we move to $\Omega^{p}$ to relax $p^{p}$ by using $\omega^{p}$ in (28). Finally, we return the updated values of $p^{p}$ to the overlap region in $\Omega^{f}$ before a new smoothing iteration can be carried out. We wish to emphasize again that this smoothing process with the data communication is implemented on each grid level in the hierarchy. There is no difference in the performance of the Uzawa smoother if we would start the relaxation method with the poroelasticity part.

Comparing the communication steps of the two smoothers, the Vanka smoother needs less communication than the Uzawa smoother. In a complete smoothing iteration on the whole domain, the Vanka smoother requires two communication phases. After the smoothing of the Stokes subsystem, the data is transferred to the overlap region. Then, the transfer is needed again when the poroelastic part is updated. Conversely, the Uzawa smoother requires four communication stages in the smoothing process since it is an equation-wise smoother. In a sequential implementation, we see no significant difference in CPU time between a Vanka smoother and an Uzawa smoother iteration. If the algorithm would be performed in parallel, the Vanka smoother could be more efficient, as the Vanka smoother requires fewer communication phases which implies less communication start-up time. Generally, a coupled smoother is often somewhat more expensive than a decoupled smoother, if we need to consider line-wise or plane-wise smoothing within a multigrid algorithm.

\subsection{Coarse-grid correction}

Regarding the discrete operators on the coarser grids in the hierarchy, direct discretization of the continuous operators is considered. At the coarsest level of the grid, a direct solver is implemented, which is easy, accurate and cheap.

Besides the smoother, other important components in multigrid are the inter-grid transfer operators. For the staggered arrangement of the unknowns, the transfer operators between two levels in the grid hierarchy are defined as follows: at displacement or velocity grid points six-point restrictions are applied and at pressure grid points one considers a four-point restriction. The restriction operators are shown in the stencil notation [15] as

$$
R_{h, 2 h}^{u}=\frac{1}{8}\left(\begin{array}{ccc}
1 & 2 & 1 \\
& * & \\
1 & 2 & 1
\end{array}\right)_{h}, R_{h, 2 h}^{v}=\frac{1}{8}\left(\begin{array}{lll}
1 & & 1 \\
2 & * & 2 \\
1 & & 1
\end{array}\right)_{h}, R_{h, 2 h}^{p}=\frac{1}{4}\left(\begin{array}{ccc}
1 & & 1 \\
& * & \\
1 & & 1
\end{array}\right)_{h},
$$


respectively. As the prolongation operators $P_{2 h, h}^{u / v / p}$, we choose the adjoints of the restrictions. The interpolation and restriction operators must be accordingly altered at boundary points or neighbors of boundary points.

In particular, the unknowns at the interface are treated as boundary points of their own subdomains. For the unknowns $v^{p}$ at the interface, the restriction operator is adapted to

$$
R_{h, 2 h}^{v^{p}}=\frac{1}{8}\left(\begin{array}{lll}
1 & & 1 \\
2 & * & 2 \\
0 & & 0
\end{array}\right)_{h},
$$

which has the same structure as those stencils for the inner grid points, but we set the weights of the points outside the poroelastic subdomain $\Omega^{p}$ to be zero. Following the same rule for the Stokes unknowns $v^{f}$ at the interface,

$$
R_{h, 2 h}^{v^{f}}=\frac{1}{8}\left(\begin{array}{lll}
0 & & 0 \\
2 & * & 2 \\
1 & & 1
\end{array}\right)_{h},
$$

is obtained as the restriction in that case.

Remark. As is commonly done, we mainly deal with the case of constant physical parameters, such as parameter $K$. However, the proposed multigrid solution method can be generalized to varying $K$-values. In [25] (and in [26]), we have performed numerical experiments for the coupled Darcy-Stokes system in which the porous medium was modeled by a random heterogeneous hydraulic conductivity $K$. When the multigrid algorithm with Uzawa smoother is applied, the optimal relaxation parameter $\omega$ is varied within the poroelastic domain, because $\omega$ depends on $K$. Additionally, the random field values should be transferred from fine to coarse grid levels in an accurate way, for an efficient multigrid method. Therefore, the corresponding suitable relaxation parameters for each grid point on each grid level should be calculated.

\section{Numerical experiments}

Three numerical experiments are presented in this section. We aim to study the accuracy of the discretization scheme, the convergence performance of the proposed multigrid method with the coupled and decoupled smoothers, and the application of the method for some test cases that may resemble realistic situations. The efficiency and robustness of the monolithic multigrid method with respect to different values of the physical parameters are also investigated.

In all numerical experiments, the initial approximation is chosen to be random, and the stopping criterion is given by,

$$
\frac{\| \text { residual } \|_{\infty}}{\| \text { right-hand side } \|_{\infty}} \leq \text { tolerance } \cdot \frac{\| \text { initial residual } \|_{\infty}}{\| \text { right-hand side } \|_{\infty}},
$$

where the tolerance is chosen to be $10^{-10}$. Moreover, for simplicity we consider uniform meshes with grid size $h$ in both directions on each subdomain. ${ }^{2}$

\subsection{Analytic test}

In the first numerical experiment, we deal with a coupled Stokes-poroelasticity problem with a known analytic solution on the domain $\Omega=(0,1) \times(0,2)$. The domain is divided into a free flow part $\Omega^{f}=(0,1) \times(0,1)$ and a porous medium subdomain $\Omega^{p}=(0,1) \times(1,2)$ by the interface $\Gamma=(0,1) \times\{1\}$. The exact solution is chosen to be

$$
\left\{\begin{array}{l}
u^{f}=u^{p}=\left(y^{2}-y\right) e^{t}, \\
v^{f}=v^{p}=0, \\
p^{f}=p^{p}=x e^{t},
\end{array}\right.
$$

and the source terms have been subsequently determined. Dirichlet boundary conditions are imposed at the exterior boundaries of the coupled geometry. At the internal interface, equations (5)-(9), are considered. In this test, the analytic solution satisfies all interface conditions when $\mu=v$.

Errors on different grid sizes First of all, we compare the numerical solution with the given exact solution for the following values of the coefficients, $K=\lambda=\mu=v=\rho=1$. The final time is $T=1$. The errors in maximum norm for each unknown on different meshes are shown in Table 1 . As we expected, by decreasing the time step with a factor 4 and the spatial mesh width by a factor 2, second-order accuracy is confirmed for poroelasticity problem, whereas for the Stokes problem, we achieve second-order accuracy for velocities and first-order for the pressure field.

\footnotetext{
2 In [12], we applied the multigrid method for porous medium flow when anisotropies due to grid stretching appeared. It is thus straightforward to generalize the proposed method for the coupled system to an anisotropic grid setting.
} 
Table 1

Maximum norm errors of variables $u^{f / p}, v^{f / p}$ and $p^{f / p}$ for different grid sizes with parameters $K=1, \lambda=1, \mu=1, \nu=1$ and $\rho=1$.

\begin{tabular}{lllll}
\hline & Grid-size & & & \\
\cline { 2 - 5 } & $16 \times 32 \times 1$ & $32 \times 64 \times 4$ & $64 \times 128 \times 16$ & $128 \times 256 \times 64$ \\
\hline$u^{f}$ & $2.45 \times 10^{-3}$ & $7.58 \times 10^{-4}$ & $2.00 \times 10^{-4}$ & $5.04 \times 10^{-5}$ \\
$v^{f}$ & $4.22 \times 10^{-3}$ & $1.28 \times 10^{-3}$ & $3.34 \times 10^{-4}$ & $8.43 \times 10^{-5}$ \\
$p^{f}$ & $1.60 \times 10^{-1}$ & $6.99 \times 10^{-2}$ & $3.02 \times 10^{-2}$ & $1.37 \times 10^{-2}$ \\
$u^{p}$ & $2.38 \times 10^{-3}$ & $6.30 \times 10^{-4}$ & $1.62 \times 10^{-4}$ & $4.09 \times 10^{-5}$ \\
$v^{p}$ & $9.45 \times 10^{-4}$ & $2.31 \times 10^{-4}$ & $5.89 \times 10^{-5}$ & $1.49 \times 10^{-6}$ \\
$p^{p}$ & $5.64 \times 10^{-4}$ & $1.81 \times 10^{-4}$ & $4.94 \times 10^{-5}$ & $1.28 \times 10^{-5}$ \\
\hline
\end{tabular}

Table 2

Two-grid convergence factors, $\rho$, predicted by LFA for poroelastic and Stokes subproblems, separately, for different values of the parameters $K$ and $v$ and different numbers of smoothing steps, $v_{1}+v_{2}$.

\begin{tabular}{|c|c|c|c|c|c|c|}
\hline \multirow[t]{2}{*}{$v_{1}+v_{2}$} & \multicolumn{3}{|c|}{ Poroelasticity } & \multicolumn{3}{|c|}{ Stokes } \\
\hline & $K=1$ & $K=10^{-3}$ & $K=10^{-6}$ & $v=1$ & $v=10^{-3}$ & $v=10^{-6}$ \\
\hline 2 & 0.36 & 0.36 & 0.36 & 0.20 & 0.20 & 0.78 \\
\hline 3 & 0.22 & 0.22 & 0.22 & 0.09 & 0.09 & 0.51 \\
\hline 4 & 0.14 & 0.14 & 0.14 & 0.06 & 0.05 & 0.37 \\
\hline
\end{tabular}

Table 3

Asymptotic convergence factors, $\rho_{h}$, for the multigrid $W$-cycle based on Uzawa smoother for the coupled Stokes-poroelasticity problem, for different values of the physical parameters $K$ and $v$ and different numbers of smoothing steps $v_{1}+v_{2}$.

\begin{tabular}{lllllllllll}
\hline & $K$ & 1 & \multicolumn{3}{c}{$10^{-3}$} & \multicolumn{5}{c}{$10^{-6}$} \\
\cline { 2 - 9 } & $\nu$ & 1 & $10^{-3}$ & $10^{-6}$ & 1 & $10^{-3}$ & $10^{-6}$ & 1 & $10^{-3}$ & $10^{-6}$ \\
\hline$v_{1}+v_{2}$ & 2 & 0.40 & 0.40 & 0.71 & 0.40 & 0.40 & 0.72 & 0.40 & 0.40 & 0.72 \\
& 3 & 0.27 & 0.27 & 0.49 & 0.27 & 0.27 & 0.49 & 0.26 & 0.26 & 0.49 \\
& 4 & 0.23 & 0.23 & 0.36 & 0.23 & 0.23 & 0.36 & 0.22 & 0.22 & 0.36 \\
\hline
\end{tabular}

Local Fourier analysis results In this section, we confirm that the asymptotic convergence factor of the monolithic multigrid based on the Uzawa smoother for the coupled problem can be predicted by means of the worst of the two-grid convergence factors obtained by LFA for the individual poroelastic and Stokes subproblems. For different values of the hydraulic conductivity $K$ and viscosity $v$, the two-grid convergence factors predicted by LFA are presented for the poroelastic and Stokes problems separately in Table 2 . The results are obtained for different numbers of smoothing steps, $v_{1}+v_{2}$. From the table, it is observed that the multigrid method based on Uzawa smoother is robust for each subproblem.

In Table 3, the asymptotic convergence factors obtained by using the monolithic multigrid $W$-cycle together with Uzawa smoother for the coupled Stokes-poroelasticity problem are presented. Homogeneous Dirichlet boundary conditions are applied at the exterior boundaries of the coupled domain. To avoid round-off errors, a random initial guess and zero right-hand sides are chosen on a fine-grid of size $h=1 / 128$. We show the convergence factors after 100 multigrid cycles so that the asymptotic convergence rates can be reached. Comparing Table 2 and 3, we observe that these factors match the worst of the two-grid convergence factors predicted by LFA for the individual subproblems. When $v$ is small, the numerical convergence factors can be estimated by LFA at a high accuracy. The slight discrepancy for some test cases is due to the involved coupling at the interface. The discretization scheme at the interface as well as the implementation of the Uzawa smoother for the whole coupled problem lead to an efficient solver.

Comparison between different cycles Next, we investigate the efficiency of the multigrid $W$ - and $V$-cycles with different preand post-smoothing steps. We employ the Vanka as well as the Uzawa smoother in the monolithic multigrid algorithm for the coupled problem. For large values of hydraulic conductivity and viscosity, $K \approx 1, v \approx 1$, it is reported in [27,28] that decoupled iterative solution methods, like versions of domain-decomposition techniques, perform well. However it has been shown in [29] that a DD method may converge slowly on fine meshes for small values of $K$ and $v$, which resembles a tight coupling of the problems in the two systems. To test the efficiency of our numerical algorithm, realistic values of the coefficients are chosen for the test for which other methods have shown convergence difficulties. In particular $K=10^{-3}$, $v=0.035, \lambda=10^{6}$ and $\mu=2.5 \times 10^{5}$. In Table 4, the number of iterations necessary to reach the stopping criterion as well as the average multigrid convergence factors (in brackets) are shown for different smoothers, multigrid cycles and preand post-smoothing steps. The results are computed on a $64 \times 128$-grid with only one time step. As it can be seen from the table, generally highly satisfactory results are obtained for both Vanka and Uzawa smoothers. Taking into account both the convergence rates and their computational cost, the $W(2,2)$-cycle results to be fastest among the different choices. We 
Table 4

Number of iterations necessary to achieve the desired convergence (and convergence factors in brackets) by using $W$ - and $V$-cycles with different numbers of pre- and post-smoothing steps on a $64 \times 128 \times 1$-grid.

\begin{tabular}{llllll}
\hline \multicolumn{6}{l}{$K=10^{-3}, \lambda=10^{6}, \mu=2.5 \times 10^{5}, \nu=0.035$ and $\rho=1$} \\
\hline Smoother & $W(1,1)$ & $W(1,2)$ & $W(2,2)$ & $V(2,2)$ & $V(3,3)$ \\
\hline Vanka & $19(0.31)$ & $14(0.22)$ & $13(0.20)$ & $19(0.35)$ & $16(0.29)$ \\
Uzawa & $25(0.45)$ & $18(0.33)$ & $15(0.26)$ & $26(0.48)$ & $20(0.38)$ \\
\hline
\end{tabular}

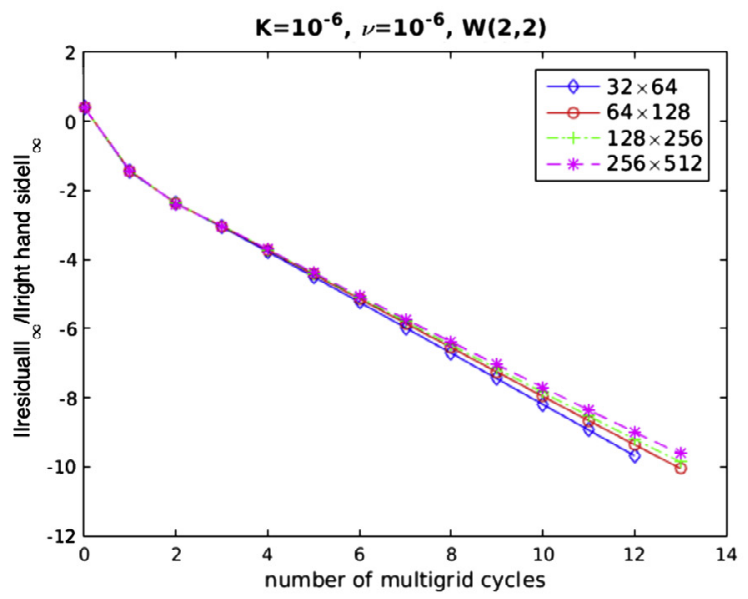

(a)

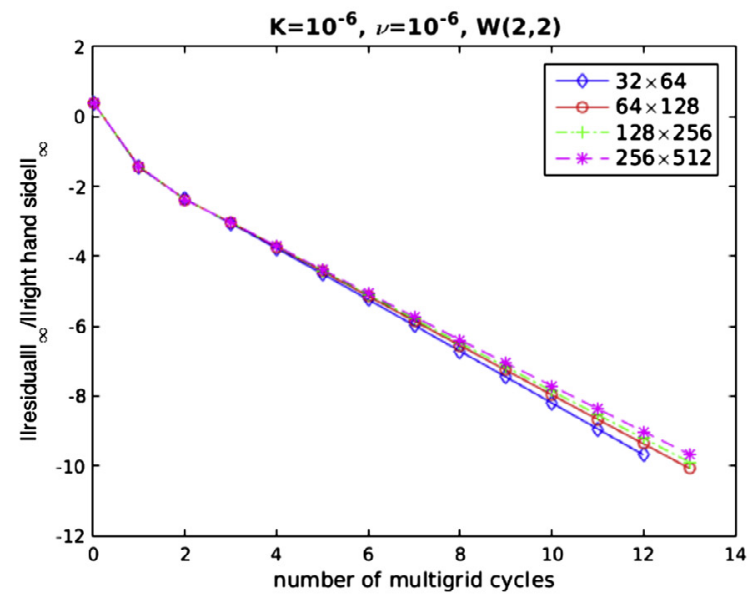

$(b)$

Fig. 6. History of the convergence of the $W(2,2)$-multigrid method for different values of the physical parameters: $(a) K=10^{-4}, v=10^{-3}$, and $(b) K=$ $10^{-6}, v=10^{-6}$.

Table 5

Convergence results with different values of time step $\tau$.

\begin{tabular}{lllll}
\hline$K=10^{-3}, \lambda=10^{6}, \mu=2.5 \times 10^{5}, \nu=10^{-3}$ and $\rho=1$ \\
\hline$\tau$ & $10^{-3}$ & $5 \times 10^{-4}$ & $2.5 \times 10^{-4}$ & $1.25 \times 10^{-4}$ \\
\hline Vanka & $16(0.20)$ & $16(0.20)$ & $16(0.20)$ & $16(0.20)$ \\
Uzawa & $19(0.28)$ & $19(0.28)$ & $19(0.28)$ & $19(0.28)$ \\
\hline
\end{tabular}

compare the CPU time of a $W(2,2)$-cycle with either the Vanka or the Uzawa smoother. For the Vanka smoother, it takes around $0.86 \mathrm{~s}$, whereas for the Uzawa smoother, it takes 1.08s. It has been implemented on a MacBook Pro with a $2.6 \mathrm{GHz}$ Intel Core i5 processor.

Multigrid convergence with different parameters We are interested in the multigrid performance with respect to different values of the physical parameters. In geoscientific applications, parameters $K$ and $v$ are typically very small numbers. It is a challenge for any solution method to deal with these small parameters and exhibit rapid convergence. In Fig. 6, for different grid sizes $h=1 / 2^{k}(k=5,6,7,8)$, the robustness of the proposed multigrid method with the Vanka smoother is displayed for different values of $K$ and $v$ and different grid sizes. The maximum norm of the residual divided by the maximum norm of the right-hand side is plotted in logarithmic scale against the number of multigrid cycles necessary to fulfill the stopping criterion. The multigrid convergence rate is independent of the physical parameters and the grid sizes, and approximately 13 iterations are needed to reduce the residual by 11 orders of magnitude for these more difficult cases.

Convergence results with different time steps Four different values of the time step $\tau=10^{-3}, 5 \times 10^{-4}, 2.5 \times 10^{-4}, 1.25 \times 10^{-4}$ are chosen to check the convergence behavior of multigrid with the two smoothers. As it can be observed from Table 5 , where the number of iterations needed to fulfill the stopping criterion together with the mean convergence rate (in brackets) are shown, the proposed method is stable and robust with the varying time step.

\subsection{Two-block realistic test}

In the next numerical test, we simulate the dynamical coupling process in a straight pipe with deformable porous media at the boundaries. To reduce the computational effort, we consider a halved domain with symmetry boundary conditions. 


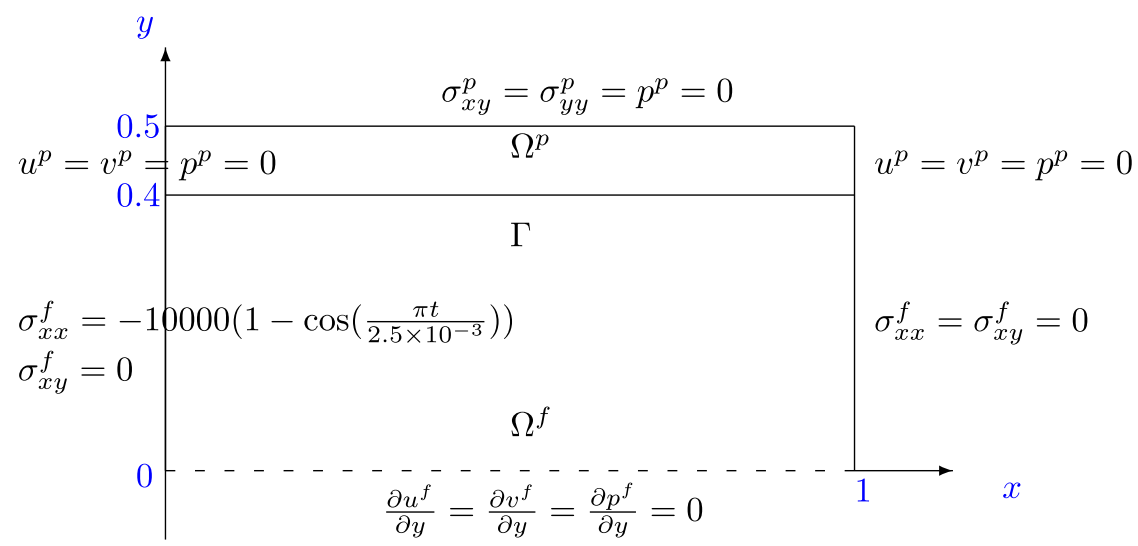

Fig. 7. Geometry of the coupled two-block Stokes-poroelasticity problem. Subdivision of the domain into a free flow subregion $\Omega^{f}$ and a porous medium subdomain $\Omega^{p}$, separated by an internal interface $\Gamma$.

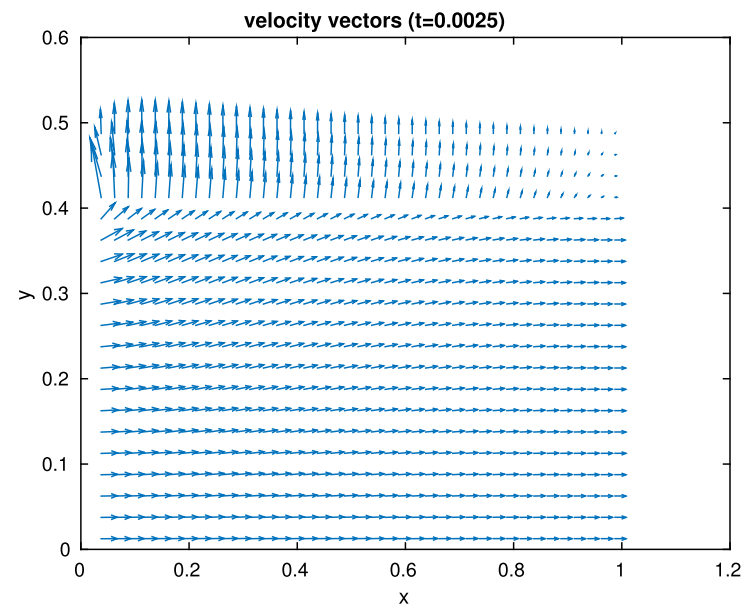

(a)

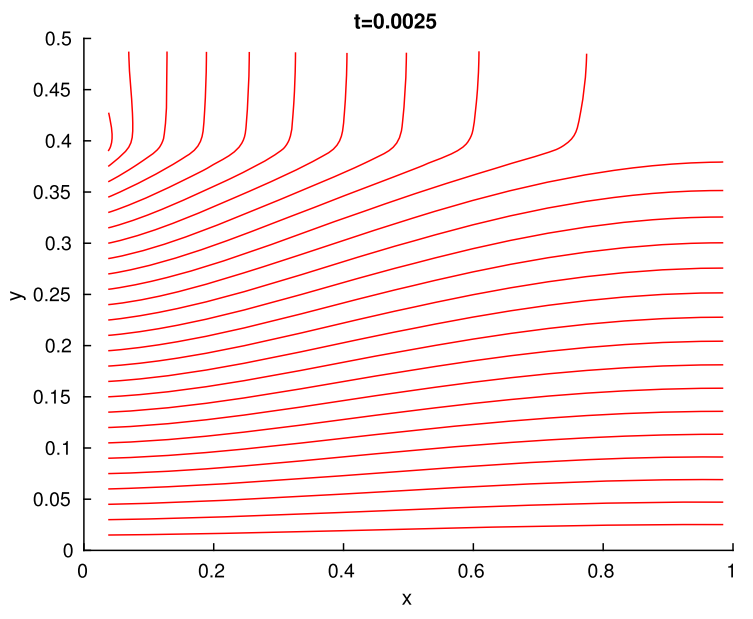

(b)

Fig. 8. For $t=0.0025$ : (a) Velocity vectors in the domain $\Omega$. For a better view, the velocity vectors in the porous medium are multiplied by 4 . (b) Corresponding streamlines of the fluid flow.

This domain for the coupled problem is depicted in Fig. 7, where $\Omega^{f}$ represents a pipe with free flow inside, while $\Omega^{p}$ is a thin porous medium layer at the top. The Neumann condition is imposed at the inlet of free flow system, while at the outlet a homogeneous Neumann condition is applied.

We take the data from the experiment in [7]. The final time is $T=0.01$ and the time step is $\tau=1.25 \times 10^{-3}$. The values of the physical parameters are chosen as $K=10^{-4}, \lambda=10^{6}, \mu=2.5 \times 10^{5}, v=0.0035$ and $\rho=1$. Within the porous medium, the fluid velocity in the pores is related to the pressure gradient and can thus be computed and visualized too. In Figs. 8 and 9, velocity vectors and corresponding streamlines of the fluid flow in the whole domain $\Omega$ are shown at two different time points, $t=0.0025$ and $t=0.005$. Since the inlet stress is periodic, we have $\sigma_{x x}^{f}=20000$ at times $t=0.0025$ and $t=0.0075$, whereas $\sigma_{x x}^{f}=0$ when $t=0.005$ and $t=0.01$. A $160 \times 80$-grid is considered as the finest grid, and a 5-level multigrid method is used in this test. By using the multigrid $W(2,2)$-cycle, a similar highly satisfactory convergence is obtained as for the first test.

For the case with the large stress value at the inlet (Fig. 8), the fluid penetrates the porous medium and we see deformation in the porous material near the interface (Fig. $10(a)$ ). The pressure solutions are presented in Fig. $10(b)$. When the inlet stress is zero (Fig. 9), the flow hardly seeps into the porous medium. Due to periodicity, at $t=2.5 \times 10^{-3}$ and $t=7.5 \times 10^{-3}$, the solutions are similar, as expected.

\subsection{Multi-block realistic test}

Here we investigate the performance of the proposed multigrid method for the coupled system for a more complex geometry. The domain of the coupled problem is shown in Fig. 11. Since the length of the free flow domain $\Omega^{f}$ and the 


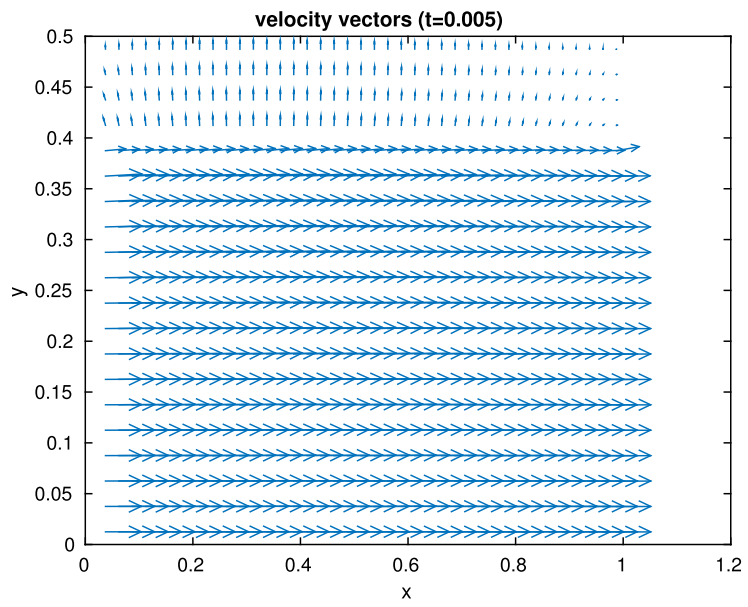

(a)

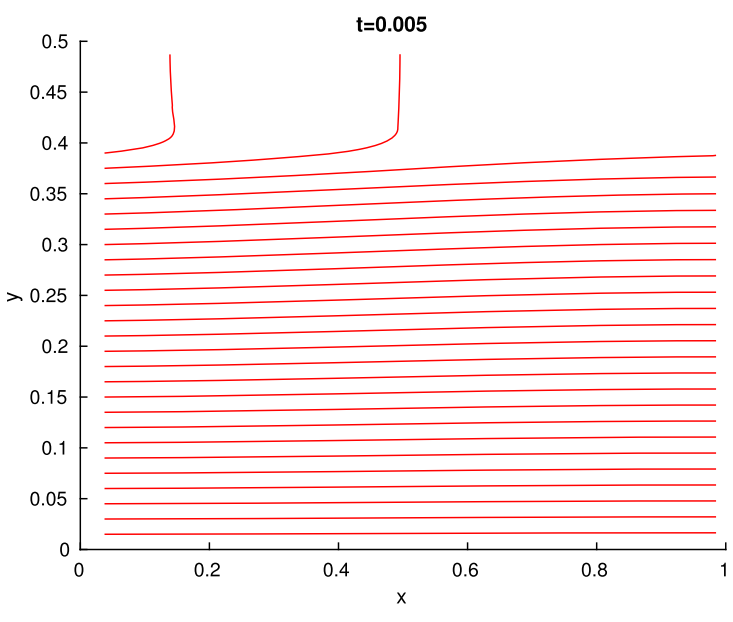

(b)

Fig. 9. For $t=0.005$ : (a) Velocity vectors in the domain $\Omega$. For a better view, the velocity vectors in the porous medium are multiplied by 4 . (b) Corresponding streamlines of the fluid flow.

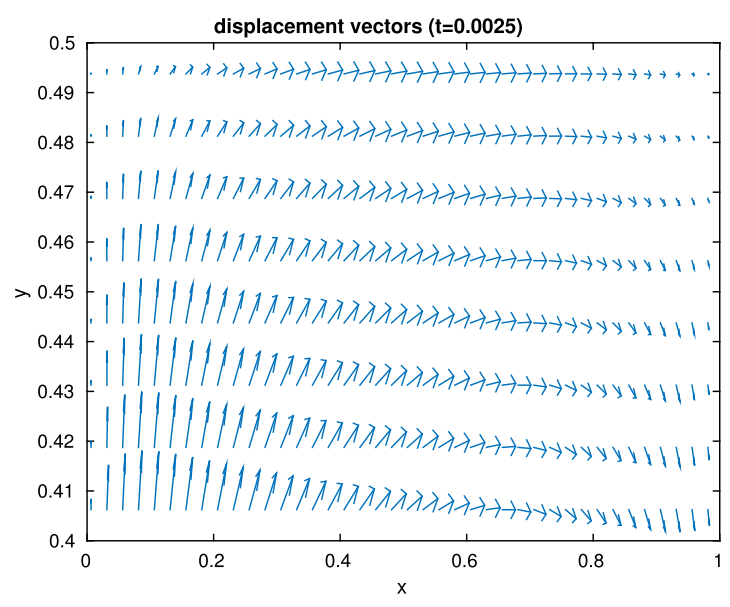

$(a)$

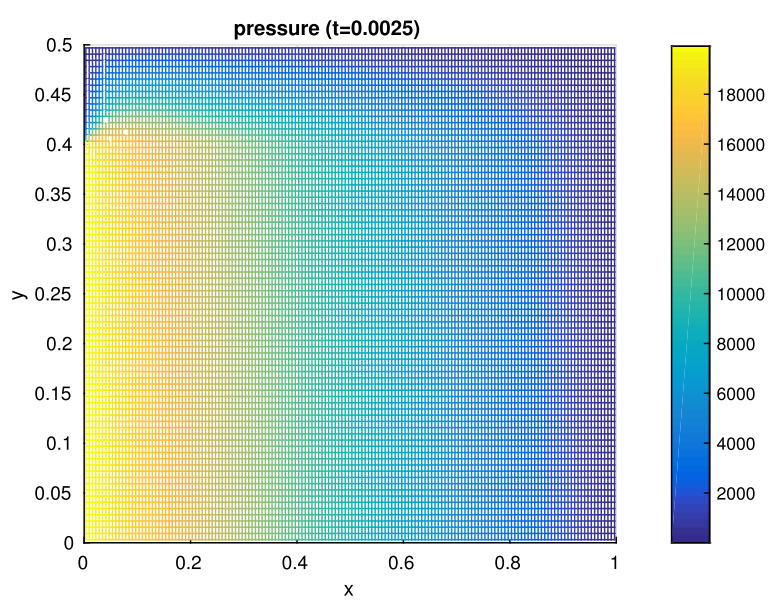

(b)

Fig. 10. For $t=0.0025$ : (a) Displacement vectors in $\Omega^{p}$, and (b) pressure in the domain $\Omega$. (For interpretation of the references to color in this figure, the reader is referred to the web version of this article.)

porous medium $\Omega^{p}$ are not identical, the domain is divided into four different blocks corresponding to the Stokes (Block1, Block2 and Block3) and porous medium (Block4) domains, respectively. A strategy similar to the one used in the previous two-block test can be straightforwardly adapted for the four block case. The information transferred between Block2 and Block4 is the same as before. For the Stokes domain, two artificial boundaries are generated by the grid partitioning approach. As communication between the subgrids in $\Omega^{f}$ is necessary, an overlap region of one cell length is created on each grid level along the artificial boundaries. The fluid inflow in $\Omega^{f}$ is specified by $\sigma_{x x}^{f}=-20000$. There is an small exit at the right-side vertical boundary of the fluid flow domain, where the stress-free boundary is imposed. The height of the exit is 0.1 . All the other boundary conditions are specified in Fig. 11. We are interested in the motion of the fluid flow under different parameter settings. In particular, we examine whether we can mimic two different conditions for the porous medium, i.e., we prescribe either permeable or impermeable conditions at the exterior boundary of $\Omega^{p}$. The values for the physical parameters (except the hydraulic conductivity $K$ ) are as in the two-block pipe flow test. The solution is computed on the target grid shown in Table 6. The numbers of iterations necessary to achieve the desired convergence by using the $W(2,2)$-cycle with both Uzawa and Vanka smoothers are very similar for all multi-block tests. For the Vanka smoother, 20 iterations are required, whereas for the Uzawa smoother we need 22 iterations. Both smoothers perform highly satisfactory. The Vanka smoother is again somewhat more robust when the physical parameters are extremely small. 


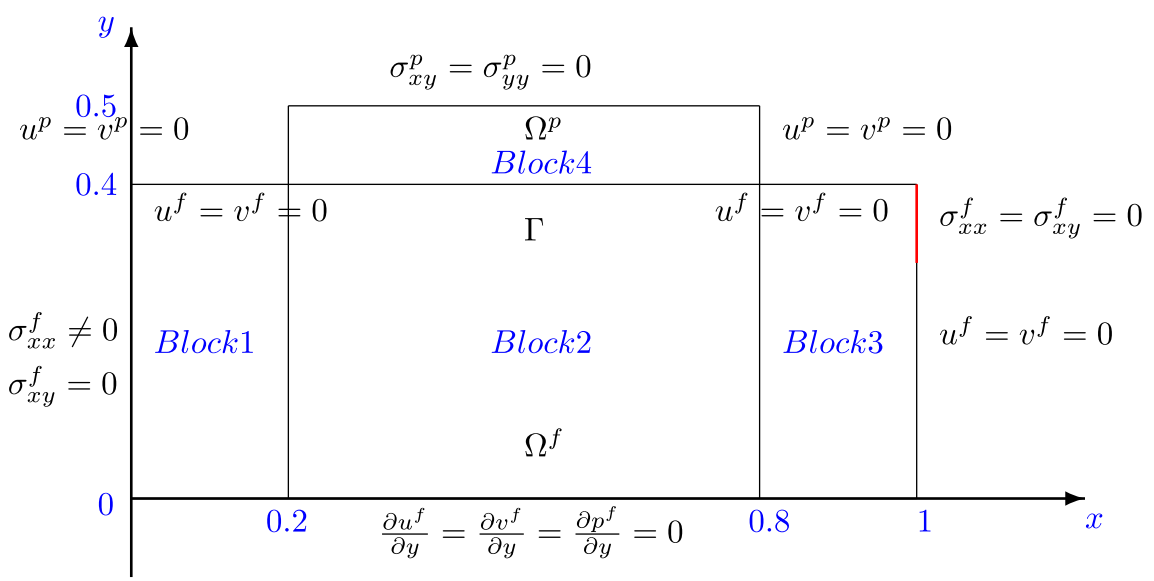

Fig. 11. Geometry of the coupled multi-block Stokes-poroelasticity problem. (For interpretation of the references to color in this figure, the reader is referred to the web version of this article.)

\section{Table 6}

Fine grid in the computational tests.

\begin{tabular}{lllll}
\hline & Block1 & Block2 & Block3 & Block4 \\
\hline Grid & $64 \times 128$ & $192 \times 128$ & $64 \times 128$ & $192 \times 32$ \\
\hline
\end{tabular}

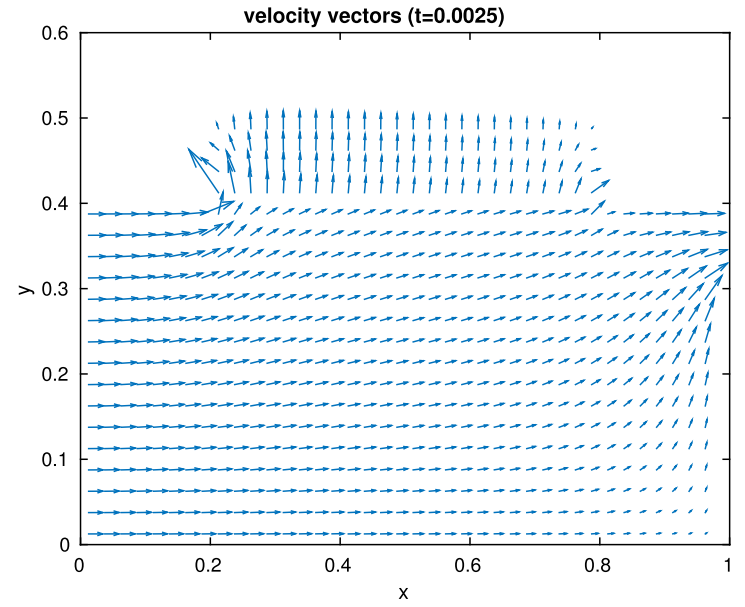

(a)

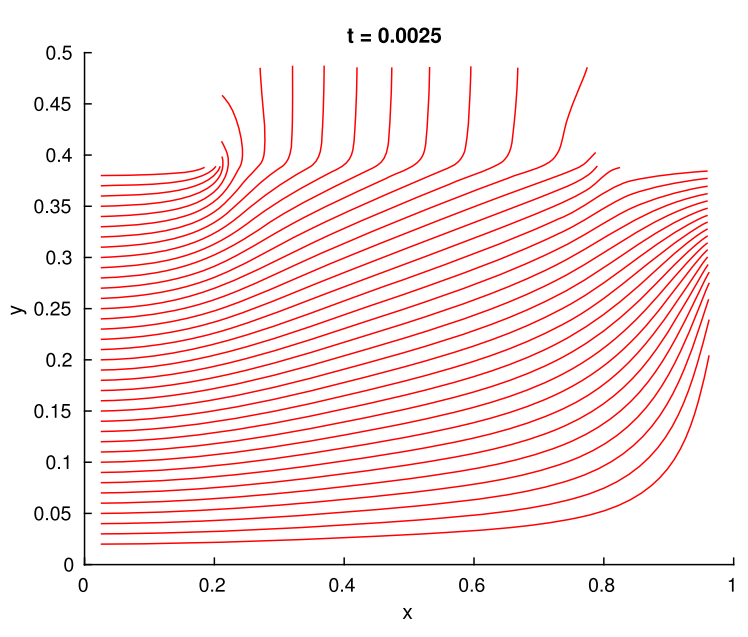

(b)

Fig. 12. For $K=10^{-4}$ and $t=0.0025$ : (a) Velocity vectors in the domain $\Omega$. For a better view, the velocity vectors in the porous medium are multiplied by 4 . (b) Corresponding streamlines of the fluid flow.

\subsubsection{Permeable conditions on the exterior boundary of $\Omega^{p}$}

We first impose drained conditions $\left(p^{p}=0\right)$ for the pressure on the exterior boundary of the porous medium. This test addresses a tangential flow filtration setting. Tangential flow filtration can be used in applications, as waste water treatment or protein purification.

We present flow fields. The velocity vectors in the computational domain, together with the streamlines are shown in Fig. 12. It is observed that at the beginning (Fig. 12) there is much flow seeping into the porous medium. The flow in the top filter then leaves the porous medium through the exterior boundary. The deformation of the porous medium is presented in Fig. 13 (a). As expected, deformation occurs at the left part of the porous medium. The pressure solutions in the domain are shown in Fig. $13(b)$.

\subsubsection{Impermeable conditions on the exterior boundary of $\Omega^{p}$}

Next, impermeable conditions are imposed on the exterior boundary of the porous medium, which can be applied in a reservoir simulation. We study the fluid motion with different values of hydraulic conductivity $K$. 


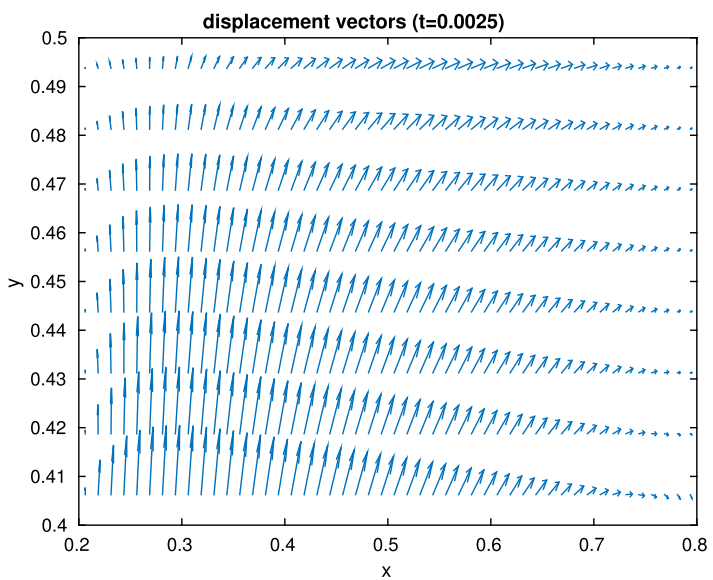

$(a)$

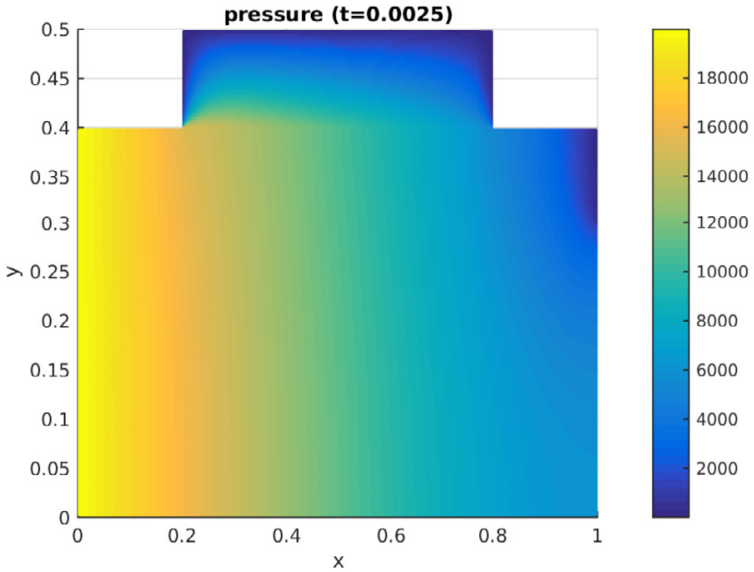

(b)

Fig. 13. For $K=10^{-4}$ and $t=0.0025$ : (a) Displacement vectors in $\Omega^{p}$, and $(b)$ pressure in the domain $\Omega$. (For interpretation of the references to color in this figure, the reader is referred to the web version of this article.)

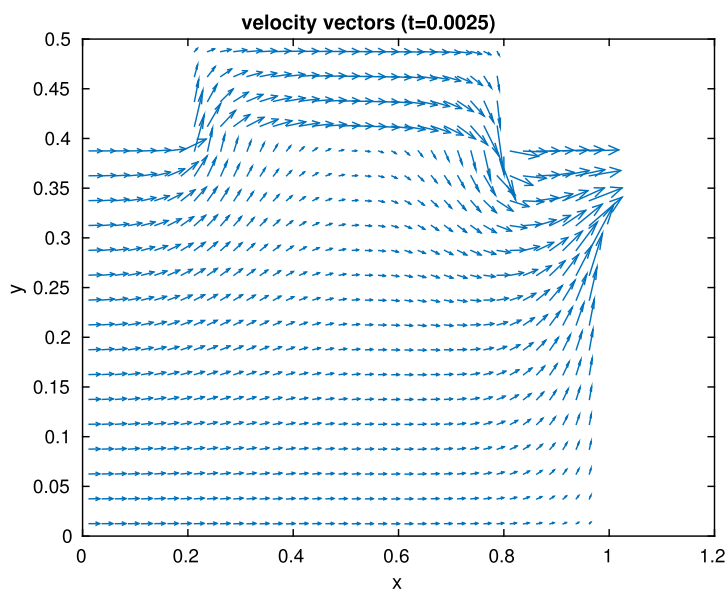

(a)

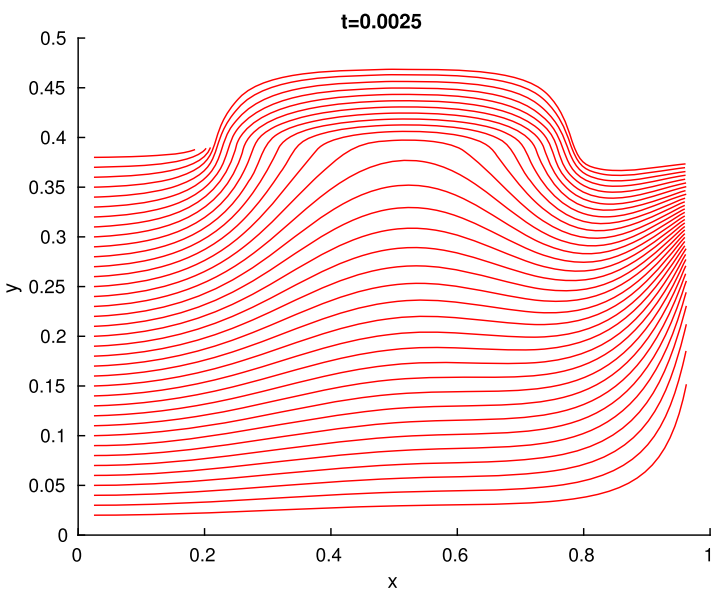

$(b)$

Fig. 14. For $K=0.01$ and $t=0.0025$ : (a) Velocity vectors in the domain $\Omega$. All vectors have been scaled by 2 . (b) Corresponding streamlines of the fluid flow.

In Fig. 14, we show the results corresponding to $K=0.01$. With such a high hydraulic conductivity of the porous medium, the free flow can easily penetrate the porous medium. The values of the velocity in $\Omega^{f}$ and $\Omega^{p}$ do not differ much. Since it is supposed there is no Darcy flow across the external boundary of $\Omega^{p}$, the fluid is forced to leave the porous medium through the interface, then flows towards the exit of the Stokes domain, see Fig. 14.

In Fig. 15, the velocity solutions corresponding to $K=10^{-4}$ are represented. Since the hydraulic conductivity of the porous medium is very low, the velocity of the Darcy flow is one to two orders of magnitude smaller compared with the velocity of the Stokes flow. A bulk of fluid goes through the exit of the channel directly. The deformation of the porous medium for smaller values of $K$ is bigger than that for higher values of $K$. The higher the hydraulic conductivity, the smaller resistance of the porous medium to the fluid flow and thus, the smaller the stresses are. Lower stresses imply smaller deformation or displacements.

\section{Conclusions}

In this paper, an efficient monolithic multigrid method is developed for a coupled system composed of a free fluid flow and a deformable porous medium. A model based on the Stokes equations and poroelastic equations is formulated with appropriate interface conditions. The finite volume method on a staggered grid is chosen as the discretization scheme for the coupled problem. For the variables near the interface, special discretizations are required based on the interface conditions. Note that in our work at the interface two unknowns belonging to different subsystems are defined at the 


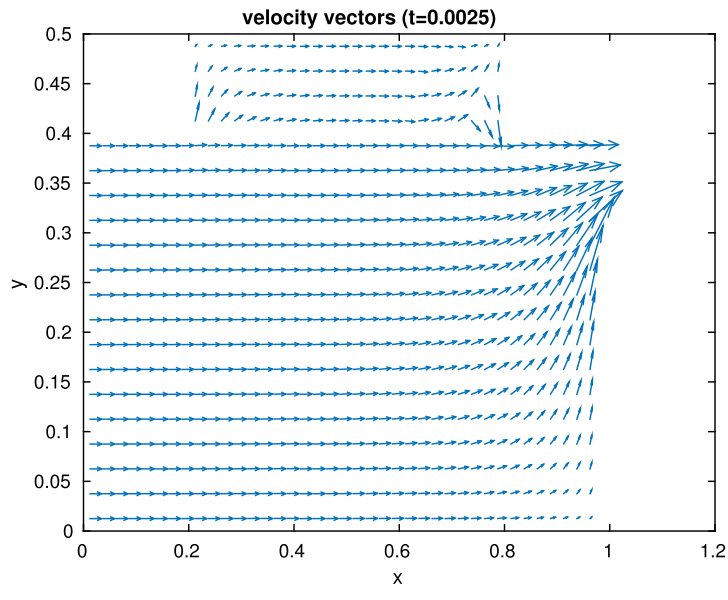

(a)

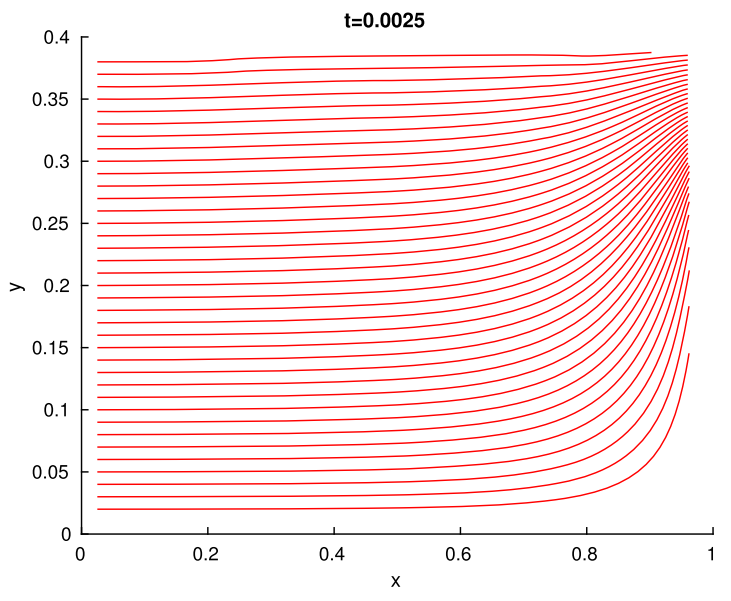

(b)

Fig. 15. For $K=10^{-4}$ and $t=0.0025$ : (a) Velocity vectors in the domain $\Omega$. For a better view, velocity vectors in the porous medium are multiplied by 18 . (b) Corresponding streamlines of the fluid flow.

same grid point. To solve the Stokes-poroelasticity system, a monolithic multigrid method with either a coupled Vanka or a decoupled Uzawa smoother is employed and these smoothers are compared. Both smoothers perform highly satisfactory for many parameter sets; only for extremely small parameters the coupled smoother exhibits a more robust convergence.

In the smoothing process, the two subsystems are coupled based on the grid partitioning technique. To achieve an excellent multigrid convergence behavior, the information is exchanged between neighboring subdomains on each grid level. Numerical experiments confirm that the discretization scheme is stable and accurate. Moreover, a highly satisfactory convergence of the monolithic multigrid method is obtained for the coupled system. Particularly, for some realistic and difficult problems where the values of the physical parameters are typically small, the proposed method performs very well.

\section{Acknowledgements}

P. Luo is grateful for the financial support by the China Scholarship Council (CSC). The research of C. Rodrigo has been partially supported by FEDER/MCYT Projects MTM2016-75139-R and the DGA (Grupo consolidado PDIE). F.J. Gaspar and C.W. Oosterlee have received funding from the European Union's Horizon 2020 research and innovation programme under the Marie Sklodowska-Curie grant agreement No. 705402, POROSOS.

\section{Appendix A. Discretization at interior points}

\section{A.1. Discretization of poroelastic equations}

Here we briefly present the staggered discretization of the poroelastic equation. We take the horizontal displacement $u_{i+\frac{1}{2}, j}^{p}$ as an example, since the discretization for the vertical component can be deduced in a similar way. The control volume $V_{i+\frac{1}{2}, j}$ in Fig. A.16 is considered. By discretizing the $x$-momentum equation, we have

$$
-\left(\frac{\left(\sigma_{x x}^{p, m}\right)_{e}-\left(\sigma_{x x}^{p, m}\right)_{w}}{h}+\frac{\left(\sigma_{x y}^{p, m}\right)_{n}-\left(\sigma_{x y}^{p, m}\right)_{s}}{h}\right)=\left(f_{1}^{p, m}\right)_{i+\frac{1}{2}, j}
$$

where $\left(\sigma_{x x}^{p, m}\right)$ and $\left(\sigma_{x y}^{p, m}\right)$ are components of the stress tensor, respectively. Approximating these components as

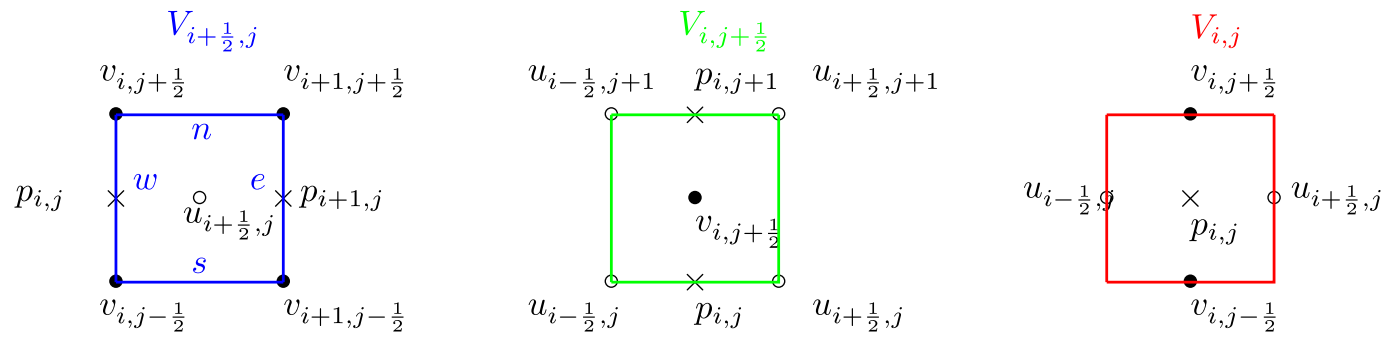

Fig. A.16. Control volumes for the primary unknowns: $u$ (left), $v$ (middle), $p$ (right), together with the corresponding indexing for each variable. 


$$
\begin{aligned}
& \left(\sigma_{x x}^{p, m}\right)_{e}=\frac{\lambda+2 \mu}{h}\left(u_{i+\frac{3}{2}, j}^{p, m}-u_{i+\frac{1}{2}, j}^{p, m}\right)+\frac{\lambda}{h}\left(v_{i+1, j+\frac{1}{2}}^{p, m}-v_{i+1, j-\frac{1}{2}}^{p, m}\right)-p_{i+1, j}^{p, m}, \\
& \left(\sigma_{x x}^{p, m}\right)_{w}=\frac{\lambda+2 \mu}{h}\left(u_{i+\frac{1}{2}, j}^{p, m}-u_{i-\frac{1}{2}, j}^{p, m}\right)+\frac{\lambda}{h}\left(v_{i, j+\frac{1}{2}}^{p, m}-v_{i, j-\frac{1}{2}}^{p, m}\right)-p_{i, j}^{p, m}, \\
& \left(\sigma_{x y}^{p, m}\right)_{n}=\frac{\mu}{h}\left(u_{i+\frac{1}{2}, j+1}^{p, m}-u_{i+\frac{1}{2}, j}^{p, m}+v_{i+1, j+\frac{1}{2}}^{p, m}-v_{i, j+\frac{1}{2}}^{p, m}\right), \\
& \left(\sigma_{x y}^{p, m}\right)_{s}=\frac{\mu}{h}\left(u_{i+\frac{1}{2}, j}^{p, m}-u_{i+\frac{1}{2}, j-1}^{p, m}+v_{i+1, j-\frac{1}{2}}^{p, m}-v_{i, j-\frac{1}{2}}^{p, m}\right),
\end{aligned}
$$

and substituting them in (A.1), we will obtain the discrete formulation.

For the pressure unknowns $p_{i, j}^{p}$, the backward Euler scheme is considered for the time-dependent term in (3). The discrete equation is obtained by discretizing the second equation in (3) over control volume $V_{i, j}$ (Fig. A.16, right side), resulting in

$$
\begin{aligned}
\frac{1}{h \tau}\left(u_{i+\frac{1}{2}, j}^{p, m}-u_{i-\frac{1}{2}, j}^{p, m}+\right. & \left.v_{i, j+\frac{1}{2}}^{p, m}-v_{i, j-\frac{1}{2}}^{p, m}\right)-\frac{1}{h \tau}\left(u_{i+\frac{1}{2}, j}^{p, m-1}-u_{i-\frac{1}{2}, j}^{p, m-1}+v_{i, j+\frac{1}{2}}^{p, m-1}-v_{i, j-\frac{1}{2}}^{p, m-1}\right) \\
& -\frac{K}{h^{2}}\left(p_{i+1, j}^{p, m}+p_{i-1, j}^{p, m}+p_{i, j+1}^{p, m}+p_{i, j-1}^{p, m}-4 p_{i, j}^{p, m}\right)=\left(f^{p, m}\right)_{i, j} .
\end{aligned}
$$

Superscripts $m$ and $m-1$ denote the current and the previous time steps respectively. The solutions at current time step $m$ can be calculated immediately from the values at the previous time.

In the case of variables located at the external boundary, where Neumann boundary conditions for the displacement are imposed, the corresponding control volumes are half the size of the inner control volumes, and are treated accordingly.

\section{A.2. Discretization of Stokes equations}

Regarding the momentum equations in (1), we here describe the discretization for the first component of the equation. By discretizing the equation over control volume $V_{i+\frac{1}{2}, j}$ (Fig. A.16, left side), the discrete equation for $u_{i+\frac{1}{2}, j}^{f}$ yields

$$
\frac{\rho}{\tau}\left(u_{i+\frac{1}{2}, j}^{f, m}-u_{i+\frac{1}{2}, j}^{f, m-1}\right)-\left(\frac{\left(\sigma_{x x}^{f, m}\right)_{e}-\left(\sigma_{x x}^{f, m}\right)_{w}}{h}+\frac{\left(\sigma_{x y}^{f, m}\right)_{n}-\left(\sigma_{x y}^{f, m}\right)_{s}}{h}\right)=\left(f_{1}^{f, m}\right)_{i+\frac{1}{2}, j} .
$$

The components of the stress tensor are approximated as

$$
\begin{aligned}
\left(\sigma_{x x}^{f, m}\right)_{e} & =-p_{i+1, j}^{f, m}+2 v \frac{u_{i+\frac{3}{2}, j}^{f, m}-u_{i+\frac{1}{2}, j}^{f, m}}{h}, \\
\left(\sigma_{x x}^{f, m}\right)_{w} & =-p_{i, j}^{f, m}+2 v \frac{u_{i+\frac{1}{2}, j}^{f, m}-u_{i-\frac{1}{2}, j}^{f, m}}{h}, \\
\left(\sigma_{x y}^{f, m}\right)_{n} & =v\left(\frac{u_{i+\frac{1}{2}, j+1}^{f, m}-u_{i+\frac{1}{2}, j}^{f, m}}{h}+\frac{v_{i+1, j+\frac{1}{2}}^{f, m}-v_{i, j+\frac{1}{2}}^{f, m}}{h}\right), \\
\left(\sigma_{x y}^{f, m}\right)_{s} & =v\left(\frac{u_{i+\frac{1}{2}, j}^{f, m}-u_{i+\frac{1}{2}, j-1}^{f, m}}{h}+\frac{v_{i+1, j-\frac{1}{2}}^{f, m}-v_{i, j-\frac{1}{2}}^{f, m}}{h}\right) .
\end{aligned}
$$

The discrete formula for the horizontal unknown $u_{i+\frac{1}{2}, j}^{f, m}$ then is obtained by substituting (A.8)-(A.11) into (A.7).

For the mass balance equation, the following discrete equation is obtained by discretizing the second equation in (1) over control volume $V_{i, j}$

$$
\frac{u_{i+\frac{1}{2}, j}^{f, m}-u_{i-\frac{1}{2}, j}^{f, m}}{h}+\frac{v_{i, j+\frac{1}{2}}^{f, m}-v_{i, j-\frac{1}{2}}^{f, m}}{h}=0 .
$$

\section{Appendix B. Derivation of optimal values for relaxation parameters}

In [17], it was proved that for the Uzawa smoother we have

$$
\mu \leq \bar{\mu}=\max \left(\left(\mu_{A}\right)^{1 / 2}, \mu_{S}\right),
$$


where $\mu$ is defined as the smoothing factor of the relaxation, $\mu_{A}$ is the smoothing factor of $M_{A}$ and $\mu_{S}$ can be interpreted as the smoothing factor of the Richardson iteration for the Schur complement,

$$
\mu_{S}:=\sup _{\Theta_{h i g h}^{2 h}} \rho\left(I-\omega\left(C+B A^{-1} B^{T}\right)\right),
$$

where $\Theta_{\text {high }}^{2 h}$ is the space of high frequencies. Details about the basis of LFA can be found in [25]. There are no essential difficulties to obtain bounds for $\mu_{A}$, see for example [30]. However, to estimate $\mu_{S}$ is somewhat involved since information about the eigenvalues of the Schur complement is needed. In particular, the bound of $\mu_{S}$ is determined by the maximum and minimum eigenvalues (denoted by $\beta_{\max }$ and $\beta_{\min }$, respectively,) on the high frequencies. Let $\zeta$ be a positive real number such that $\zeta<2$. By defining $\kappa_{\beta}=\frac{\beta_{\max }}{\beta_{\min }}$, the following bound for $\mu_{S}$ is obtained (see [17] for more details),

$$
\mu_{S} \leq \max \left(\zeta-1,1-\frac{\zeta}{\kappa_{\beta}}\right) .
$$

Note that the choice of $\zeta<2$ is to ensure that $\mu_{S}<1$. Then, by choosing a value of $\zeta$ to minimize the expression in (B.3), we obtain an optimal relaxation parameter for the Uzawa smoother as follows

$$
\omega=\frac{2}{\beta_{\max }+\beta_{\min }} .
$$

\section{Appendix C. Details of communication}

In Fig. C.17, the two subgrids are extended by adding "one cell" overlap regions that are drawn in dashed lines. The red circles, dots and crosses represent the unknowns of the Stokes system, while the blue circles, dots and crosses denote the
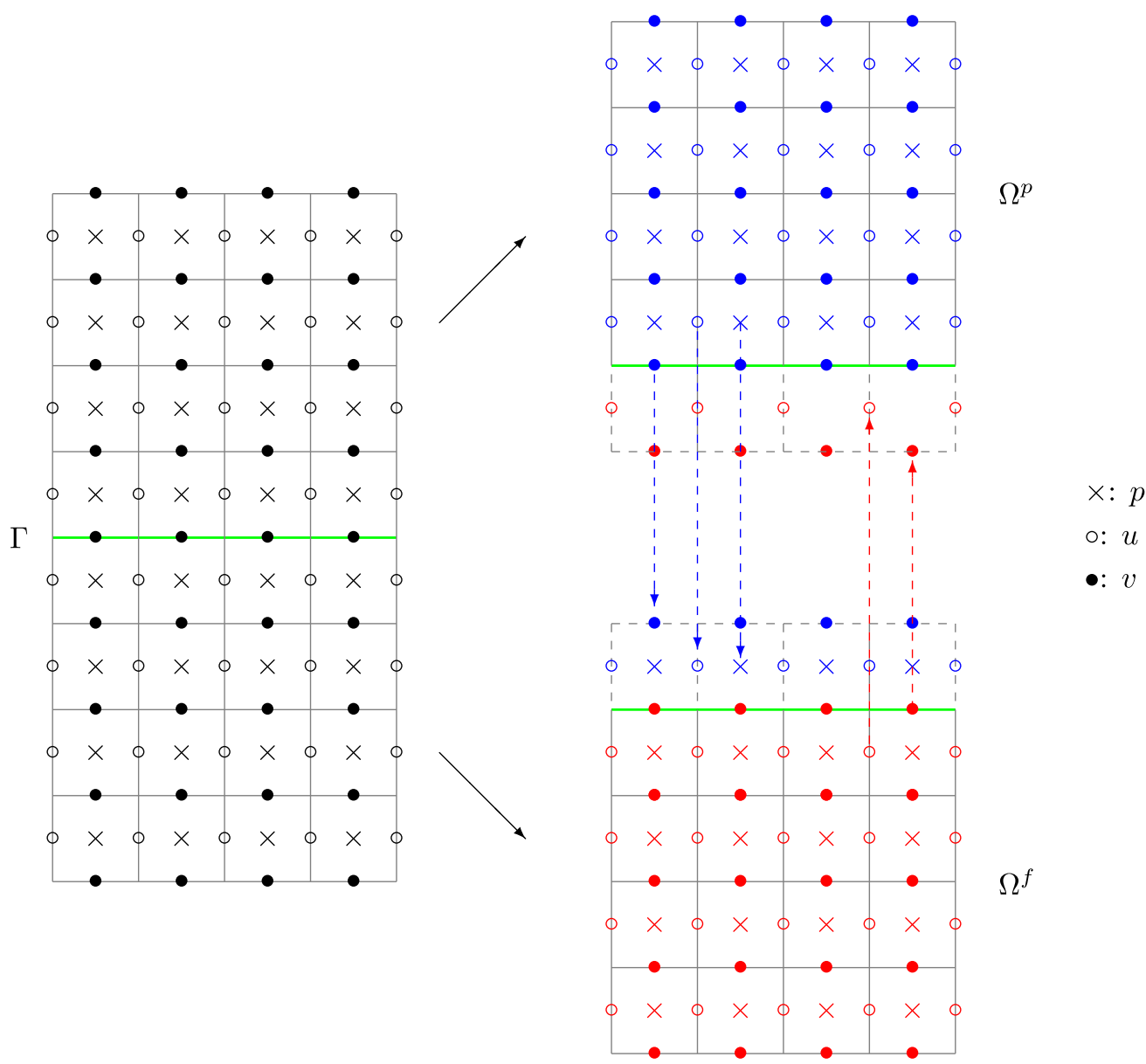

Fig. C.17. Communications between two partitioned subgrids. (For interpretation of the references to color in this figure, the reader is referred to the web version of this article.) 
poroelastic unknowns. In the Stokes subdomain $\Omega^{f}$, for example, the color of the unknowns in the overlap region (blue) is different from the color of those in $\Omega^{f}$ (red). Since $u^{p}, v^{p}$ and $p^{p}$ appear in the discrete formula for the Stokes velocity $v^{f}$ at the interface, the values of these three poroelastic unknowns are transferred to the extra region of the Stokes domain, represented by blue arrows in Fig. C.17. For the poroelastic part, the transfer strategy is the same. The difference is that only velocities $u^{f}$ and $v^{f}$ are needed in the special discretizations for the poroelastic unknowns. So, the fluid pressure $p^{f}$ is not transferred to the overlap region in $\Omega^{p}$. The communication from the Stokes subsystem to the poroelastic subsystem is indicated by the red arrows in Fig. C.17.

\section{References}

[1] M. Biot, General theory of three-dimensional consolidation, J. Appl. Phys. 12 (2) (1941) 155-164.

[2] M. Biot, Theory of elasticity and consolidation for a porous anisotropic solid, J. Appl. Phys. 26 (2) (1955) $182-185$.

[3] R. Showalter, Poroelastic Filtration Coupled to Stokes Flow, Lecture Notes in Pure and Applied Mathematics, vol. 242, 2005 , pp. 229-241.

[4] M. Benzi, G. Golub, J. Liesen, Numerical solution of saddle point problems, Acta Numer. 14 (1) (2005) 1-137.

[5] S. Muntz, Fluid Structure Interaction for Fluid Flow Normal to Deformable Porous Media, Ph.D. thesis, Department of Mathematics, Technical University of Kaiserslautern, Kaiserslautern, 2008.

[6] S. Patankar, Numerical Heat Transfer and Fluid Flow, CRC Press, 1980.

[7] S. Badia, A. Quaini, A. Quarteroni, Coupling Biot and Navier-Stokes equations for modelling fluid-poroelastic media interaction, J. Comput. Phys. 228 (21) (2009) 7986-8014.

[8] A. Mikelić, I. Yotov, R. Zakerzadeh, P. Zunino, Partitioning strategies for the interaction of a fluid with a poroelastic material based on a Nitsche's coupling approach, Comput. Methods Appl. Mech. Eng. 292 (2015) 138-170.

[9] M. Bukač, I. Yotov, P. Zunino, An operator splitting approach for the interaction between a fluid and a multilayered poroelastic structure, Numer. Methods Partial Differ. Equ. 31 (4) (2015) 1054-1100.

[10] S. Vanka, Block-implicit multigrid solution of Navier-Stokes equations in primitive variables, J. Comput. Phys. 65 (1) (1986) 138-158.

[11] J. Maitre, F. Musy, P. Nigon, A fast solver for the Stokes equations using multigrid with a Uzawa smoother, in: Advances in Multi-Grid Methods, Springer, 1985, pp. 77-83.

[12] P. Luo, C. Rodrigo, F. Gaspar, C. Oosterlee, On an Uzawa smoother in multigrid for poroelasticity equations, Numer. Linear Algebra Appl. 24 (2017) e2074, http://dx.doi.org/10.1002/nla.2074.

[13] F. Gaspar, F. Lisbona, C. Oosterlee, A stabilized difference scheme for deformable porous media and its numerical resolution by multigrid methods, Comput. Vis. Sci. 11 (2) (2008) 67-76.

[14] F. Gaspar, F. Lisbona, C. Oosterlee, R. Wienands, A systematic comparison of coupled and distributive smoothing in multigrid for the poroelasticity system, Numer. Linear Algebra Appl. 11 (2-3) (2004) 93-113.

[15] C. Oosterlee, F. Gaspar, Multigrid relaxation methods for systems of saddle point type, Appl. Numer. Math. 58 (12) (2008) 1933-1950.

[16] U. Trottenberg, C. Oosterlee, A. Schuller, Multigrid, Academic Press, 2000.

[17] F. Gaspar, Y. Notay, C. Oosterlee, C. Rodrigo, A simple and efficient segregated smoother for the discrete Stokes equations, SIAM J. Sci. Comput. 36 (3) (2014) A1187-A1206.

[18] A. Brandt, Multi-level adaptive solutions to boundary-value problems, Math. Comput. 31 (138) (1977) 333-390.

[19] M. Perić, R. Kessler, G. Scheuerer, Comparison of finite-volume numerical methods with staggered and collocated grids, Comput. Fluids 16 (4) (1988) $389-403$

[20] F. Gaspar, F. Lisbona, P. Vabishchevich, Staggered grid discretizations for the quasi-static Biot's consolidation problem, Appl. Numer. Math. 56 (6) (2006) $888-898$

[21] F. Harlow, J. Welch, Numerical calculation of time-dependent viscous incompressible flow of fluid with free surface, Phys. Fluids 8 (12) (1965) 2182-2189.

[22] B. Lamichhane, B. Wohlmuth, Mortar finite elements for interface problems, Computing 72 (3) (2004) $333-348$.

[23] O. Axelsson, Iterative Solution Methods, Cambridge University Press, 1996.

[24] A. Brandt, N. Dinar, Multigrid solutions to elliptic flow problems, Numer. Methods Partial Differ. Equ. (1979) 53-147.

[25] P. Luo, C. Rodrigo, F. Gaspar, C. Oosterlee, Uzawa smoother in multigrid for the coupled porous medium and Stokes flow system, SIAM J. Sci. Comput. 39 (5) (2017) S633-S661, https://doi.org/10.1137/16M1076514.

[26] P. Kumar, P. Luo, F. Gaspar, C. Oosterlee, A multigrid multilevel Monte Carlo method for transport in Darcy-Stokes system, 2017, submitted for publication.

[27] M. Hoffmann, The Navier-Stokes-Darcy Problem, Bachelor thesis, Weierstrass Institute, Germany, 2013.

[28] A. Caiazzo, V. John, U. Wilbrandt, On classical iterative subdomain methods for the Stokes-Darcy problem, Comput. Geosci. 18 (5) (2014) 711-728.

[29] M. Discacciati, A. Quarteroni, A. Valli, Robin-Robin domain decomposition methods for the Stokes-Darcy coupling, SIAM J. Numer. Anal. 45 (3) (2007) $1246-1268$.

[30] R. Wienands, W. Joppich, Practical Fourier Analysis for Multigrid Methods, CRC Press, 2004 